\title{
STEADY STATE SOLUTIONS IN A MODEL OF A CHOLESTERIC LIQUID CRYSTAL SAMPLE ${ }^{\sharp}$
}

\author{
FERNANDO P. DA COSTA*, MICHAEL GRINFELD, NIGEL J. MOTTRAM, \\ JOÃO T. PINTO, AND KEDTYSACK XAYXANADASY
}

\begin{abstract}
Motivated by recent mathematical studies of Fréedericksz transitions in twist cells and helix unwinding in cholesteric liquid crystal cells $[3,4,16,18]$, we consider a model for the director configuration obtained within the framework of the Frank-Oseen theory and consisting of a nonlinear ordinary differential equation in a bounded interval with non-homogeneous mixed boundary conditions (Dirichlet at one end of the interval, Neumann at the other). We study the structure of the solution set using the depth of the sample as a bifurcation parameter. Employing phase space analysis techniques, time maps, and asymptotic methods to estimate integrals, together with appropriate numerical evidence, we obtain the corresponding novel bifurcation diagram and discuss its implications for liquid crystal display technology. Numerical simulations of the corresponding dynamic problem also provide suggestive evidence about stability of some solution branches, pointing to a promising avenue of further analytical, numerical, and experimental studies.
\end{abstract}

\section{INTRODUCTION}

1.1. Motivation: cholesteric liquid crystals. The strong anisotropic nature of the constitutive molecules in a liquid crystal, and their preference to align with each other, lead to a local macroscopic orientational order [6]. This orientational order allows us to define an axis of local rotational symmetry, the average molecular orientation at location $\mathbf{x}=\left(x_{1}, x_{2}, x_{3}\right)$. This local symmetry axis defines a macroscopic variable, and is usually represented by a unit vector $\mathbf{n}=\mathbf{n}(\mathbf{x})$, called the director. The director may vary with the location $\mathbf{x}$ in space as a result of internal or external influences, to create director distortion structures that may change the stored elastic energy of the system. In this paper we consider liquid crystals that

Date: Published online: October 28, 2020.

2010 Mathematics Subject Classification. Primary 34B15, 34C23; Secondary 41A60, 76A15.

Key words and phrases. Non-homogeneous two-points boundary value problems; Bifurcations; Asymptotic evaluation of integrals; Cholesteric liquid-crystal cells; Fréedericksz transition; Nonlinear pendulum.

Acknowledgements: FPdC and JTP were partially funded by FCT/Portugal through project CAMGSD UID/MAT/04459/2020. FPdC acknowledges financial support provided by the University of Strathclyde David Anderson Research Professorship. This work started while FPdC was staying at the Department of Mathematics of the Faculty of Natural Sciences of the National University of Laos, Vientiane, Laos, with the support of an Erasmus Mundus Mobility with Asia grant EMMA ID 2601. The hospitality of NUOL is gratefully acknowledged. Part of this work was undertaken while NJM was employed at the University of Strathclyde. We thank R. Sasportes for his help with the figures.

* Corresponding author.

\# Published in Afrika Matematika, https://doi.org/10.1007/s13370-020-00851-9. The final publication is available at link.springer.com. 
have only orientational order, termed nematics, and we do not consider those liquid crystals that also show positional ordering, which are termed smectics.

In nematic liquid crystals consisting of chiral molecules, often called cholesterics or chiral nematics, the molecular alignment may produce a macroscopic helical structure, in which the director twists along an axis in space, in either a left- or right-handed sense and with a pitch that is material- and temperature-dependent. If the cholesteric liquid crystal is enclosed in a region $\Omega \in \mathbb{R}^{3}$, the elastic free energy of the director field can be described, in the framework of the Frank-Oseen theory, by the integral

$$
W_{E}=\int_{\Omega} w_{\mathrm{FO}}(\mathbf{n}, \nabla \mathbf{n}) d \mathbf{x}
$$

where the Frank-Oseen energy density is given by [23, p. 24]

$$
w_{\mathrm{FO}}(\mathbf{n}, \nabla \mathbf{n})=\frac{1}{2}\left(K_{1}(\nabla \cdot \mathbf{n})^{2}+K_{2}(\mathbf{n} \cdot \nabla \times \mathbf{n}+q)^{2}+K_{3}\|\mathbf{n} \times \nabla \times \mathbf{n}\|^{2}\right),
$$

where $K_{1}, K_{2}, K_{3} \geqslant 0$ are the Frank elastic moduli [23, p. 21], and $q$ is the wave number associated with the intrinsic pitch of the liquid crystal helical arrangement, and thus is a measure of chirality [23, p. 25].

Liquid crystals respond to applied electric or magnetic fields, through a dielectric or diamagnetic response, by realigning their molecules in order to reduce the electromagnetic energy. In many situations, in particular when the liquid crystal is held between solid surfaces, there exists a threshold value of the applied field, above which realignment due to the dielectric or diamagnetic effect occurs. This phenomenon, called the Fréedericksz transition [23], is the basis of almost all technological applications of liquid crystal materials, including the switching behaviour of Liquid Crystal Displays. For a field F, the director-dependent electromagnetic energy due to the applied field is

$$
W_{F}=-\int_{\Omega} \frac{1}{2} \chi(\mathbf{F} \cdot \mathbf{n})^{2} d \mathbf{x}
$$

where $\chi$ is a measure of the anisotropy of the dielectric or diamagnetic susceptibility, dependent on the electromagnetic characteristics of the liquid crystal.

In the present situation we consider a layer of cholesteric liquid crystal, occupying the region $\Omega=\mathbb{R}^{2} \times(0, d)$, with the $x_{3}$ coordinate axis oriented such that $x_{3} \in(0, d)$. We assume homogeneity in the $x_{1}$ and $x_{2}$ directions so that the only independent variable is $x_{3}$, and also that the director remains in the $\left(x_{1}, x_{2}\right)$-plane throughout the region. This last assumption is justified, and often used, since in the system we describe below there are no internal and external forces acting in the $x_{3}$-direction that would perturb the director from the $\left(x_{1}, x_{2}\right)$-plane. The director field can then be written as $\mathbf{n}\left(x_{3}\right)=\left(\cos \varphi\left(x_{3}\right), \sin \varphi\left(x_{3}\right), 0\right)$, so that $\varphi\left(x_{3}\right)$ is the angle between the director and the $x_{1}$-axis. We will also assume that the orienting field is applied orthogonal to $x_{3}$, in other words in the plane in which the director lies, such that $\mathbf{F}=(0, F, 0)$. The orientation of the field with respect to the plane in which the director lies is therefore the same as in the well known twist Fréedericksz cell [23] and for the In-Plane Switching display used for many portable and touch screen devices [11]. In general, the field strength $F$ will be a function of $x_{3}$, and should be determined through the application of the appropriate Maxwell's equations. However, in the case of magnetic fields the diamagnetic susceptibility is small, 
so that the effects of director distortion do not significantly affect the local field strength, and the field strength is often assumed constant, i.e. independent of $x_{3}$ [23]. The situation for electric fields is different, with the constant electric field approximation only valid when the dielectric anisotropy is very small and often only for certain homogeneous director configurations [23]. For non-homogeneous director configurations, and in some cases even for homogeneous director configurations $[1,7,10]$, a spatially dependent electric field must be considered in order to correctly model the director-field interaction. In this paper we consider only the magnetic field situation and therefore use the approximation of a constant field strength $F$.

For this form of $\mathbf{n}$ and $\mathbf{F}$ the total free energy (per unit length in the $x_{1}$ and $x_{2}$ directions) is then given by

$$
W=W_{E}+W_{F}=\frac{1}{2} \int_{0}^{d}\left[K_{2}\left(\frac{d \varphi}{d x_{3}}-q\right)^{2}-\chi F^{2} \sin ^{2} \varphi\right] d x_{3} .
$$

As well as the effects of elasticity and an applied magnetic fields, the surfaces bounding a region may also exert an influence on the liquid crystal director. Depending on the surface material, and whether the surface has been chemically or mechanically treated, different forms of surface anchoring of the director are possible. In the present situation we assume that surface in the plane $x_{3}=0$ has been chemically and mechanically treated so as to force the liquid crystal director to have a fixed alignment in the $\left(x_{1}, x_{2}\right)$-plane, so that the boundary condition is $\varphi(0)=-\varphi_{0}$. In standard liquid crystal devices this is often termed infinite or strong planar rubbed anchoring of the director and is achieved by mechanical rubbing of a polymer coated surface. We also assume that the surface in the plane $x_{3}=d$ is not chemically or mechanically treated, but that the steric interaction of the surface and the liquid crystal molecules leads to the molecules lying in the plane of the surface. The natural boundary condition, arising from the variational minimisation of the total energy, is then $d \varphi / d x_{3}-q=0$ at $x_{3}=d$. This type of boundary condition is most commonly encountered at an untreated polymer surface, but may also be present at a free surface, where the liquid crystal is exposed to an ambient atmosphere.

The Euler-Lagrange equation for the free energy (3) is

$$
2 K_{2} \frac{d^{2} \varphi}{d x_{3}^{2}}+\chi F^{2} \sin 2 \varphi=0,
$$

which will be solved subject to the boundary conditions

$$
\begin{aligned}
& \varphi(0)=-\varphi_{0}, \\
& \frac{d \varphi}{d x_{3}}(d)-q=0 .
\end{aligned}
$$

It is worth pointing out here that the chirality of the liquid crystal does not enter equation (4) and only appears, through $q$, in the boundary condition (6). This is due to the fact that the term $K_{2} q d \varphi / d x_{3}$ in the expanded form of (3) is a null Lagrangian, i.e. it does not enter the Euler-Lagrange equation in the interior of the region. Constant solutions of equation (4) occur when $\varphi=n \pi / 2, n \in \mathbb{Z}$ and, from (3), we see that minimisers of the magnetic energy will occur for odd values of $n$. This is as to be expected since the director orientation in the bulk (i.e., away from the boundaries) preferring to align parallel or antiparallel to the 
magnetic field direction. It is the effects of chirality and confinement, which enter through the boundary conditions, that will mean complete alignment with the field is impossible.

The study of magnetic field effects in cholesterics has a long history, dating back to experimental studies in the 1960s (c.f. [19]), and equations related to (4)-(6) have been since investigated in many different situations, but were first considered by de Gennes [5] and, independently, by Meyer [17]. These first works considered an infinite region of a cholesteric of positive diamagnetic susceptibility, with the helical axis aligned perpendicular to the applied magnetic field, as in our situation. Upon increasing the magnetic field it was found that the pitch of the helix increased, with regions aligned to the electric field becoming larger, between which were $180^{\circ}$ twist reorientation regions. In an infinite region the "unwinding" of the helix, so that the helical pitch becomes infinite, occurs at a critical field strength $F=(\pi q / 2) \sqrt{K_{2} / \chi}$. This situation is discussed in detail in [6, Section 6.2.2.3]. In a finite geometry the helical pitch changes through discrete jumps in pitch length, and elastic energy [8]. The theoretical investigation in [8] has more recently been extended by a number of authors (cf. $[2,12,16,20,24])$ through the use of different boundary conditions and analysis methods. The most relevant previous work for the present situation is probably that of Kiselev and Slucking [13] and McKay [16] both of whom consider cholesteric unwinding in finite regions and with weak anchoring, which in the case of [13] is different at the two boundaries. In our situation the boundaries exhibit infinite anchoring (the Dirichlet condition at $x_{3}=0$ ) and zero anchoring (the natural, or Neumann condition at $x_{3}=d$ ) but as we show below, we see both preferred alignment angles and an increase in the pitch of the helix due to the magnetic field as in previous work.

It is convenient to non-dimensionalize the equations using the scalings

$$
\begin{aligned}
L & :=\frac{d}{2} \sqrt{\frac{\chi F^{2}}{2 K_{2}}}, \\
t & :=x_{3} \sqrt{\frac{\chi F^{2}}{2 K_{2}}}-L, \\
\phi & :=\varphi_{0}, \\
\phi^{\star} & :=q \sqrt{\frac{2 K_{2}}{\chi F^{2}}},
\end{aligned}
$$

The scaling factor used in equations (7), (8) and (10) is related to the magnetic coherence length $\xi_{m}=\sqrt{K_{2} /\left(\chi F^{2}\right)}[23$, p. 68], which is the lengthscale over which competing magnetic and elastic effects will reorient the director which is also the lengthscale of the $180^{\circ}$ reorientation regions in the helix unwinding process mentioned above. The magnetic coherence length, the layer thickness $d$ and the chiral pitch $2 \pi / q$ are the three lengthscales in this system, and the non-dimensional parameters that enter the problem above, $\phi^{\star}$ and $L$, are then ratios of these lengthscales.

If we now set

$$
x(t):=\varphi\left(x_{3}\right),
$$


we obtain the nonlinear pendulum equation

$$
x^{\prime \prime}(t)+\sin 2 x(t)=0, \quad t \in(-L, L),
$$

subject to the boundary conditions

$$
x(-L)=-\phi, \quad x^{\prime}(L)=\phi^{\star} .
$$

The system therefore contains three parameters: $\phi$, associated with the prescribed director angle at the boundary $t=-L ; \phi^{\star}$, which is related to the chirality through $q$, as well as a ratio of elastic and electromagnetic influences; and $L$, which is proportional to the original layer thickness $d$, as well as the ratio of elastic and electromagnetic influences.

The general problem (12)-(13) is a challenging multi-parameter problem and, in what follows, we analyse a restricted version of the problem. We will fix both $\phi$ and $\phi^{\star}$ but vary $L$ as a bifurcation parameter. From equations (8)-(11) we see that this situation is equivalent to keeping all parameters constant while varying the layer thickness $d$. Furthermore, to simplify computations, we take $\phi>0$; and choose a particular value of $\phi^{\star}$, which will be specified below.

The study of boundary value problems such as (12)-(13), leading to the determination of the number of solutions and their properties (including their stability properties when they are seen as equilibria of the time-dependent partial differential equation of which (12) is the stationary equation), has potentially important technological consequences. In particular, when such a liquid crystal layer is used as a display device, the device thickness $d$ is a crucially important parameter that determines its optical characteristics. The existence of multiple stable solutions can critically affect display performance and device manufacturers often prefer to have a single stable director configuration, or, in case of multiple stable solutions, one that has a significantly lower energy than all the others.

1.2. The model to be studied. In recent papers $[3,4]$ equation (12) with nonhomogeneous Dirichlet boundary value conditions, coming from a model of an achiral nematic liquid crystal cell, was considered and the structure of the set of stationary solutions when the parameter $L$ is changed was studied. As mentioned above, here we study the problem (12)-(13), $\phi>0$, where one of the boundary conditions is Neumann. In general, the parameters $\phi$ and $\phi^{\star}$ can vary independently, for instance by varying the surface director angle $\varphi_{0}$ and the chirality $q$. However, to gain insight into this problem we will assume these conditions are related in a particular way (see below). The general case will be treated elsewhere ${ }^{1}$.

The problem that will be considered is the existence of solutions to

$$
\begin{aligned}
& \left\{\begin{array}{l}
x^{\prime}=y \\
y^{\prime}=-\sin 2 x,
\end{array}\right. \\
& x(-L)=-\phi, \quad y(L)=\phi^{\star} .
\end{aligned}
$$

\footnotetext{
${ }^{1}$ Preliminary results, [15], seem to show that the analysis will follow the same lines as presented here and the results will be similar, with the main difference being the destruction of the transcritical bifurcation points at $T^{*}, T_{j}^{*}$ in the diagram of Fig. 11 in a way similar to what happened in [4] when compared with [3].
} 
where we choose

$$
\phi^{\star}:=\sqrt{1-\cos 2 \phi}
$$

This constraint on the boundary conditions may, at first sight, seem rather restrictive. In dimensional terms it is equivalent to $F=\sqrt{2 K_{2}(1-\cos 2 \varphi) /\left(\chi q^{2}\right)}$. We see, therefore, that such a constraint is equivalent to tuning the magnitude of the magnetic field to a specific value, something that can readily be achieved experimentally. This relationship between the two boundary conditions introduces symmetries in the solutions, as the case of Dirichlet boundary conditions studied in [3], but it is a reasonable first step towards the understanding of the case of general $\phi^{\star}$.

Since (14) is a system of first order ODEs, it is convenient to use the language of dynamical systems and call $t$ the time variable (although, in the context of the liquid crystal model described above, $t$ is related to the distance through the liquid crystal film.) The phase portrait of (14) is shown in Figure 1.

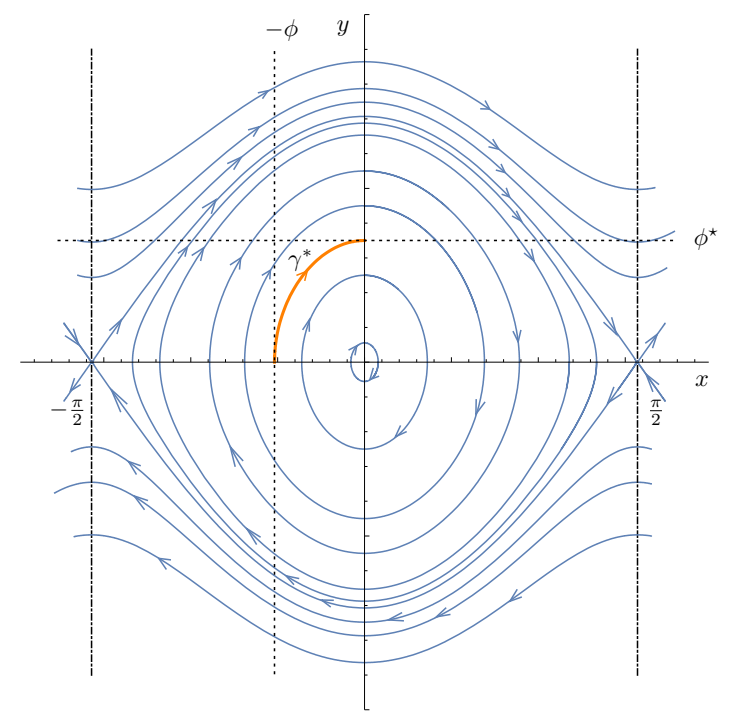

FIguRE 1. Phase portrait of the orbits of equation (14), the boundary conditions (15) to be considered, and the orbit $\gamma^{*}$ referred to in the text. The lines $x=-\frac{\pi}{2}$ and $x=\frac{\pi}{2}$ are also indicated.

System (14) has a first integral given by

$$
V(x, y)=y^{2}-\cos 2 x
$$

and from the choice of $\phi^{\star}$ we see that $V(-\phi, 0)=V\left(0, \phi^{\star}\right)$ and we conclude that the points $(-\phi, 0)$ and $\left(0, \phi^{\star}\right)$ lie on the same orbit of $(14)$.

The tools used in this paper are based on appropriately defined time maps, i.e., functions measuring the "time" $t$ spent by a given orbit of (14) between two of its points. According to what will be most appropriate for the computations, we will identify an orbit either by the ordinate of its first intersection with the $x$-axis, the $y$-axis, or the line $x=-\phi$, leading to different, although equivalent, time maps. 
We study the bifurcation diagram of solutions to (14)-(15) using the following procedure: we start by identifying a segment of an orbit of $(14), \gamma^{*}$, such that the corresponding solution, in addition to satisfying the boundary conditions (15), $x(-L)=-\phi$ and $y(L)=\phi^{\star}$, also satisfies $y(-L)=0$ and $x(L)=0$ (see Figure 1). We call the solution corresponding to $\gamma^{*}$ a critical solution (and $\gamma^{*}$ a critical (segment of an) orbit). Calling this solution critical is justified as we will prove that in bifurcation diagrams parameterized by $L$, there is more than one solution branch passing through it. The (segment of) orbit $\gamma^{*}$ corresponds to a critical time $T^{*}$, and a corresponding critical value of $L=L^{*}=T^{*} / 2>0$.

We then perturb this (segment of) orbit and investigate how the time it takes a solution to travel from one boundary condition to the other changes relative to $T^{*}$. This time is measured by appropriately defined time maps, whose definition arises naturally from the phase plane portrait and the first integral (16) (see, e.g. [14, $22])$. This approach was used in $[3,4]$ for the study of (14) with non-homogeneous Dirichlet boundary conditions; its application in the present case leads to some unexpected difficulties and the analytical study has to be completed with numerical simulations providing solid evidence for a conjecture about the existence of a single minimum of the time maps of some of the solution branches.

\section{TIME MAPS: DEFINITION AND BASIC RESUltS}

Let $\gamma$ be an orbit of the dynamical system defined by (14) passing through points $\left(x_{0}, y_{0}\right)$ and $\left(x_{1}, y_{1}\right)$. If we consider a solution of (14) that passes through $\left(x_{0}, y_{0}\right)$ at time $t=0$ and reaches $\left(x_{1}, y_{1}\right)$ at time $T$, we say below that $T$ is the time it takes $\gamma$ to travel from $\left(x_{0}, y_{0}\right)$ to $\left(x_{1}, y_{1}\right)$.

For every $\alpha \in\left(0, \frac{\pi}{2}\right)$, the orbit $\gamma_{\alpha}$ of (14) that intersects the $x$-axis at $(-\alpha, 0)$ is periodic. The time taken from the point of intersection of $\gamma_{\alpha}$ with the negative- $x$ semi-axis, $(-\alpha, 0)$, to the first intersection with the positive- $y$ semi-axis, occurring at the point $(0, \sqrt{2} \sin \alpha)$, can be computed in a standard way $[14,22]$ using the first equation of (14) and the first integral (16). The resulting function, measuring the time as a function of $\alpha$, is called a time map:

$$
T(\alpha):=\int_{0}^{\alpha} \frac{1}{\sqrt{\cos 2 x-\cos 2 \alpha}} d x .
$$

It should be noted that the function $T(\alpha)$, defined in (17), may be be written as

$$
T(\alpha)=\operatorname{am}^{-1}(\alpha, \sqrt{2 /(1-\cos (2 \alpha))}) / \sqrt{1-\cos (2 \alpha)},
$$

where $\mathrm{am}^{-1}$ is the inverse Jacobi amplitude function.

We will also need to measure the time taken by $\gamma_{\alpha}$, described above, between its point of intersection with the positive- $y$ semi-axis, $(0, \sqrt{2} \sin \alpha)$, and the point of its first intersection with the vertical line $x=\nu$, with $\nu \in\left(0, \frac{\pi}{2}\right)$. In the same way as above, the fact that (16) is a first integral allows us to conclude that this time is given by the time map

$$
T_{1}(\alpha, \nu):=\int_{0}^{\nu} \frac{1}{\sqrt{\cos 2 x-\cos 2 \alpha}} d x .
$$

Observe that $T_{1}(\alpha, \alpha)=T(\alpha)$. 
The following result is well known and will be repeatedly used below. A proof can be found in [3].

Proposition 1. Let $0<\phi<\alpha<\frac{\pi}{2}$. The time maps $T$ and $T_{1}$ defined by (17) and (18), respectively, satisfy:

(1) $\alpha \mapsto T(\alpha)$ is strictly increasing, and converges to $+\infty$ as $\alpha \rightarrow \frac{\pi}{2}$ and to $\frac{\pi}{2 \sqrt{2}}$ as $\alpha \rightarrow 0$.

(2) $\alpha \mapsto T_{1}(\alpha, \phi)$ is strictly decreasing.

To study the orbits located above the homoclinic orbit to $\left(\frac{\pi}{2}, 0\right) \equiv\left(-\frac{\pi}{2}, 0\right)$ in the positive- $y$ semi-plane, we use as an identifying parameter its intersection with some positive line (instead of the parameter $\alpha$ above, which in these cases is nonexistent since these orbits do no intersect the $x$-axis). In $[3,4]$ the ordinate $\beta$ of the intersection of the orbit with the positive- $y$ semi-axis was used as a parameter. Here we shall use the value $z:=y(-L)^{2}$ as a parameter, i.e., the square of the intersection of the orbit with the vertical line $x=-\phi$, or, in terms of the original boundary value problem, the square of the value of the derivative of the solution $x(t)$ at the boundary point $t=-L$. For orbits intersecting the $x$-axis we can easily relate the parameters $\alpha$ and $z$ using the first integral $(16): V(-\alpha, 0)=V(-\phi, \sqrt{z})$. In order not to overload the notation we shall use the same symbols, $T$ or $T_{1}$, for the time maps independently of which variable, $\alpha$ or $z$, is being used in the parameterization of the orbits.

\section{Phase space analysis of orbits bifurcating From $\gamma^{*}$}

Let $\gamma^{*}$ be the segment of orbit of (14)-(15) shown in Figure 1, with $T^{*}=2 L^{*}$ the time taken by this orbit to travel between the two boundary conditions. This segment is a subset of the periodic orbit of (14) intersecting the negative $x$-axis at $x=-\phi$. Slightly perturbing this periodic orbit to another whose intersection with the negative $x$-axis is at $-\alpha<-\phi$, with $\alpha-\phi$ sufficiently small, we easily conclude from the phase portrait and from the continuous dependence of solutions of ODEs on the initial data over finite time intervals, that there exists four distinct orbits satisfying (14)-(15) for appropriately chosen values of $L$ close to $L^{*}$. We shall denote these as solutions of type $I, A, B$, and $C$, as illustrated in Figure 2.

Taking into account the time maps defined in section 2, the time it takes each solution of the above types to travel between the two boundary conditions is given by, respectively,

$$
\begin{aligned}
T_{I}(\alpha, \phi) & :=T_{1}(\alpha, \phi)-T_{1}\left(\alpha, x^{\star}\right), \\
T_{A}(\alpha, \phi) & :=2 T(\alpha)-\left(T_{1}(\alpha, \phi)+T_{1}\left(\alpha, x^{\star}\right)\right), \\
T_{B}(\alpha, \phi) & :=T_{1}(\alpha, \phi)+T_{1}\left(\alpha, x^{\star}\right), \\
T_{C}(\alpha, \phi) & :=2 T(\alpha)-\left(T_{1}(\alpha, \phi)-T_{1}\left(\alpha, x^{\star}\right)\right),
\end{aligned}
$$

where $x^{\star}$ is the positive solution of $V\left(x^{\star}, \phi^{\star}\right)=V(-\alpha, 0)$, i.e.,

$$
x^{\star}=x^{\star}(\alpha, \phi):=\frac{1}{2} \arccos (1-\cos 2 \phi+\cos 2 \alpha) .
$$

From the phase plane portraits in Figures 1 and 2 we conclude that solutions of type $A$ and $C$ can be continued down to $-\alpha \downarrow-\frac{\pi}{2}$, which corresponds to their initial point converging to points on the homoclinic orbit to $\left(-\frac{\pi}{2}, 0\right) \equiv\left(\frac{\pi}{2}, 0\right)$ in $\{y<0\}$, but not further down: if the initial point gets to, or below, this homoclinic orbit 


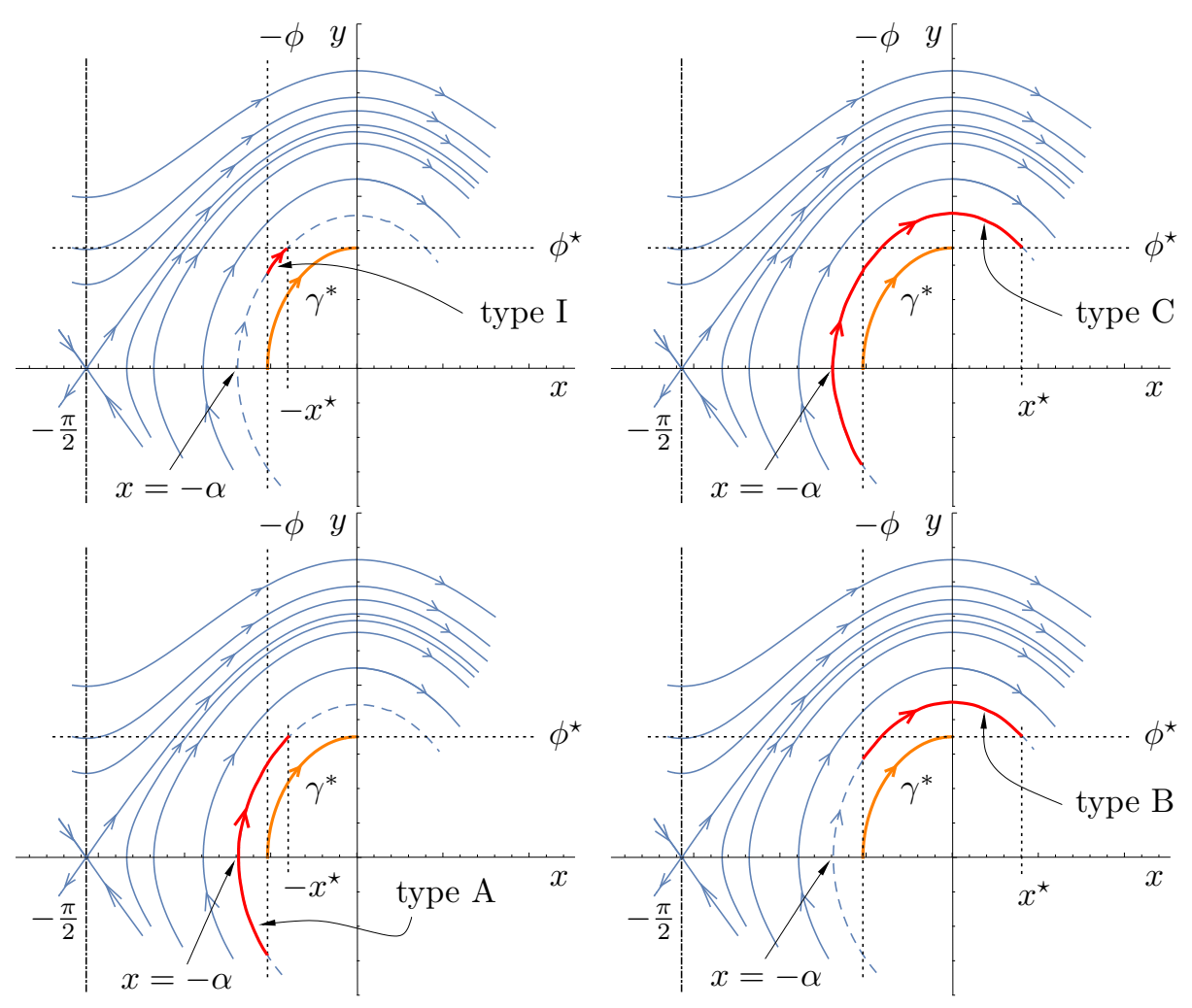

Figure 2. Phase plane illustration of the four types of orbits of (14)-(15), denoted by $A, B, C$, and $I$, obtained by a perturbation of the orbit $\gamma^{*}$

the corresponding solution remains in $\{y<0\}$, and the solution will not satisfy the boundary condition $y(L)=\phi^{\star}>0$, for any value of $L$.

In contradistinction with these cases, in principle there is no obstruction to solutions of types $I$ and $B$ to be continued above the homoclinic orbit to $\left(-\frac{\pi}{2}, 0\right) \equiv$ $\left(\frac{\pi}{2}, 0\right)$ in $\{y>0\}$. To properly handle this possibility it is convenient to parameterize the solutions, and the corresponding time maps, not by $\alpha$ but by either the ordinate of its intersection with the positive $y$-axis, $\beta$, or by the ordinate of its initial point $y(-L)$, or, as we shall do in section 4.4 and mentioned above, by the square of this quantity $z=y(-L)^{2}$. Using these parameterizations, the variable $\alpha$ in the function $x^{\star}$ needs to be correspondingly changed to $\beta, y(-L)$, or $z$, which is easily done using the fact that $V$ is a first integral to relate the various parameters, $V(-\alpha, 0)=V(0, \beta)=V(-\phi, y(-L))=V(-\phi, \sqrt{z})$, leading to the corresponding expressions for $x^{\star}$. One that we shall frequently use in what follows is the expression in terms of $z$ :

$$
\bar{x}^{\star}(z):=\frac{1}{2} \arccos (1-z)
$$


Notwithstanding the possibility of these solutions to be continued above the homoclinic, they cannot be continued for arbitrarily large values of $y(-L)$, although for different reasons, as we shall see below.

Type $I$ solutions obviously cease to exist when $y(-L)=\phi^{\star}$, since, when this occurs, the initial and final points of the solution coincide (see Figure 3.) The

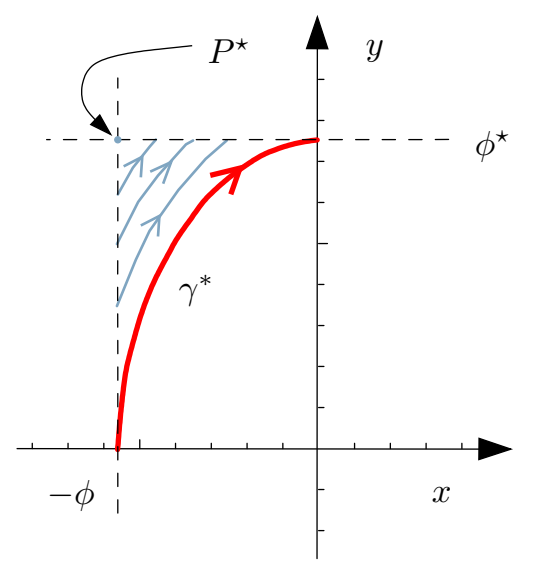

Figure 3. Continuation of type $I$ solutions of (14)-(15) for the initial point of the solution with increasing values of $y(-L)$, showing that, when $y(-L) \rightarrow \phi^{\star}$, these orbits converge to a single point $P^{\star}=\left(-\phi, \phi^{\star}\right)$ and then vanish.

reason why these solutions cannot be continued above this value of $y(-L)$ is easy to understand from the phase plane portrait: since the initial and final points of type $I$ orbits are always regular points of the phase plane, having $y(-L)$ approach the limiting value $\phi^{\star}$ we have that type $I$ solutions take less and less time $2 L$, with $L \rightarrow 0$ as $y(-L) \rightarrow \phi^{\star}$, and thus (14)-(15) make no sense in the limit. In fact, analysis of the time maps tell us exactly the same thing: taking $z \rightarrow\left(\phi^{\star}\right)^{2}$ in (19) (with the variable $z$ instead of $\alpha$ ) and noting that, by (24) and the definition of $\phi^{\star}, \lim _{z \rightarrow\left(\phi^{\star}\right)^{2}} \bar{x}^{\star}(z)=\phi$, it immediately follows that $T_{I}(z) \rightarrow 0$. In section 4.1 we will discuss the monotonicity of $T_{I}$.

The situation for type $B$ solutions is more interesting. Since all solutions of (14) above the orbit homoclinic to $\left(-\frac{\pi}{2}, 0\right) \equiv\left(\frac{\pi}{2}, 0\right)$ in $\{y>0\}$ have an absolute minimum at $x=\frac{\pi}{2}$, there are no type $B$ solutions on an orbit of (14) if the $y$ component of that minimum is bigger than $\phi^{\star}$, since in this case no segment of the orbit (and in particular no type $B$ solutions) can satisfy the boundary condition $y(L)=\phi^{\star}$. Using the first integral $V$ this means that the largest value of $y(-L)$ that a type $B$ solution can satisfy is given by $V(-\phi, y(-L))=V\left(\frac{\pi}{2}, \phi^{\star}\right)$, and so, from $\phi^{\star}=\sqrt{1-\cos 2 \phi}$, we must have $y(-L)=\sqrt{2}$, independently of $\phi$. This implies that solutions of type $B$ only exist for $y(-L) \in(0, \sqrt{2}]$.

To understand what is going on in this case, we observe that, due to the periodicity of the vector field, for initial points $(-\phi, y(-L))$ with $y(-L)$ bigger than the ordinate of the point on the homoclinic orbit (but less than $\sqrt{2}$ ), there is another solution with end point $\left(-x^{\star}, \phi^{\star}\right)$. This solution belongs to a new class of solutions 
which we shall call type $B^{\prime}$. See Figure 4 . When $y(-L) \rightarrow \sqrt{2}$ the two end points

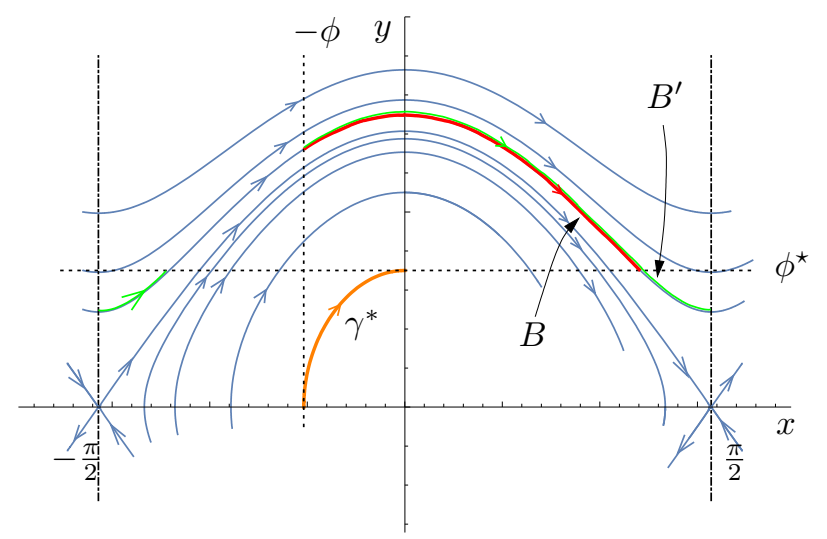

Figure 4. An orbit of (14)-(15) of type $B$ above the homoclinic orbit and the orbit of type $B^{\prime}$ with the same $y(-L)$.

of solutions of types $B$ and $B^{\prime}$ converge to one another and at $y(-L)=\sqrt{2}$ the two coincide with the point $\left(\frac{\pi}{2}, \phi^{\star}\right)$, and both cease to exist for $y(-L)>\sqrt{2}$.

\section{Bifurcation Diagram of orbits Bifurcating From $\gamma^{*}$}

To draw the bifurcation diagram of solutions bifurcating from $\gamma^{*}$ we need to put together the information in section 3 , gathered from the phase plane portrait, with information about the time spent by each solution, obtained from the study of the time maps, which we will do next.

4.1. Behavior of the type $I$ solution branch. We first consider solutions of type I. From (19), the definition of the time maps (17) and (18), and Proposition 1, we conclude that

$$
T_{I}(\alpha, \phi)=T_{1}(\alpha, \phi)-T_{1}\left(\alpha, x^{\star}\right)<T_{1}(\alpha, \phi)<T_{1}(\phi, \phi)=T(\phi)=T^{*}=2 L^{*} .
$$

Thus, in the bifurcation diagram plotted using the time spent by the orbit as the bifurcation parameter, type $I$ branch of solutions exist to the left of the bifurcation point $T^{*}$ correspondent to the critical orbit segment $\gamma^{*}$. Let us compute the derivative $\partial T_{I} / \partial \alpha$. From

$$
\begin{aligned}
\frac{\partial T_{I}}{\partial \alpha}(\alpha, \phi)= & \frac{\partial T_{1}}{\partial \alpha}(\alpha, \phi)-\frac{\partial T_{1}}{\partial \alpha}\left(\alpha, x^{\star}\right)-\frac{\partial T_{1}}{\partial x^{\star}} \frac{d x^{\star}}{d \alpha} \\
= & -\int_{x^{\star}(\alpha)}^{\phi} \frac{\sin 2 \alpha}{(\cos 2 x-\cos 2 \phi)^{\frac{3}{2}}} d x \\
& -\frac{2 \sin 2 \alpha}{\sqrt{\cos 2 x^{\star}(\alpha)-\cos 2 \phi} \sqrt{1-(1-\cos 2 \phi-\cos 2 \alpha)^{2}}},
\end{aligned}
$$

we conclude that $\partial T_{I} / \partial \alpha<0$, since it is clear from the definition of type $I$ solutions that we always have $x^{\star}<\phi$ (see Figure 2). This means that the branch of type $I$ solutions in the bifurcation diagram has no turning points.

The above computations were done using the parameterization of orbits by the parameter $\alpha$, and thus the corresponding solutions are inside the region bounded by 
the homoclinics. This is always the case when the point $\left(-\phi, \phi^{\star}\right)$ is in this region. When it is outside this region, the solutions can still be continued, as explained in section 3 , and the results above still hold using a parameterization of the orbits by either of the parameters introduced therein, namely $y(-L), z$, or $\beta$.

The results above and the discussion in section 3 allows us to conclude that the type $I$ solution branch continues monotonically to $T=0$, as shown qualitatively in Figure 5.

4.2. Behavior of the type $C$ solution branch. Consider now solutions of type $C$. From (22), the definition of the time maps (17) and (18), and Proposition 1, we conclude that

$$
\begin{aligned}
T_{C}(\alpha, \phi) & =2 T(\alpha)+T_{1}\left(\alpha, x^{\star}\right)-T_{1}(\alpha, \phi) \\
& =T(\alpha)+\underbrace{T_{1}\left(\alpha, x^{\star}\right)}_{>0}+\underbrace{\left(T(\alpha)-T_{1}(\alpha, \phi)\right)}_{>0} \\
& >T(\alpha) \\
& >T(\phi)=T^{*}=2 L^{*} .
\end{aligned}
$$

On the other hand, since by (19) and (22) we have $T_{C}(\alpha, \phi)=2 T(\alpha)-T_{I}(\alpha, \phi)$, we conclude that

$$
\frac{\partial T_{C}}{\partial \alpha}(\alpha, \phi)=2 T^{\prime}(\alpha)-\frac{\partial T_{I}}{\partial \alpha}(\alpha, \phi)>0,
$$

where the positivity comes from Proposition 1 and the result in section 4.1. Note that orbits of type $C$ are always inside the region bounded by the homoclinics and so this analysis is enough to conclude that, like the branch of type $I$ solutions, the type $C$ solution branch do not have turning points and, from Proposition 1.(1), exists globally when $L \rightarrow+\infty$, since type $C$ orbits take progressively longer times as $\alpha \rightarrow \pi / 2$.

4.3. Local behavior of the type $B$ solution branch. In this section we study the behavior of the solution branch of type $B$ solutions locally close to the bifurcation point.

Consider the branch of bifurcating solutions of (14)-(15) denoted by $B$ in section 3. As already observed, the time taken by a solution of type $B$ is given by

$$
T_{B}(\alpha, \phi)=T_{1}(\alpha, \phi)+T_{1}\left(\alpha, x^{\star}(\alpha)\right),
$$

where $T_{1}$ is the time map defined by (18) and $x^{\star}$ is defined by (23). Please see the plot of a type $B$ solution in Figure 2 in order to clarify this notation.

Since type $B$ solutions can be continued above the homoclinic orbit to $\left(-\frac{\pi}{2}, 0\right) \equiv$ $\left(\frac{\pi}{2}, 0\right)$ in $\{y>0\}$, it is natural to consider solutions parameterized by the ordinate of one of its points. It turns out that, from the computational point of view, the appropriate parameter is $z=y(-L)^{2}$. Using $V(-\phi, \sqrt{z})=V(-\alpha, 0)$, we can obtain an expression for $T_{B}$ from (21) when the solution is bounded by the homoclinics and extended to larger values of $z$ as explained in section 2. We thus have

(26) $T_{B}(z, \phi)=\int_{0}^{\phi}(z-\cos 2 \phi+\cos 2 x)^{-1 / 2} d x+\int_{0}^{\bar{x}^{\star}(z)}(z-\cos 2 \phi+\cos 2 x)^{-1 / 2} d x$

where $z \in[0,2], \phi \in\left(0, \frac{\pi}{2}\right)$, and $\bar{x}^{\star}(z)$ is defined in (24). 
4.3.1. Computations of $\frac{\partial T_{B}}{\partial z}$. We want to prove that for $z$ sufficiently close to zero $T_{B}$ satisfies $T_{B}(z, \phi)<T_{B}(0, \phi)$. To achieve this, we prove, in Proposition 4 , that $\frac{\partial T_{B}}{\partial z}(0)=-\infty$, which obviously implies the inequality.

Proposition 2. $\frac{\partial T_{B}}{\partial z}(z, \phi) \longrightarrow-\infty$ as $z \rightarrow 0$.

Proof. Differentiating (26) with respect to $z$ we get

$$
\begin{aligned}
2 \frac{\partial T_{B}}{\partial z}(z, \phi)= & -\int_{0}^{\phi}(z-\cos 2 \phi+\cos 2 x)^{-3 / 2} d x \\
& -\int_{0}^{\bar{x}^{\star}(z)}(z-\cos 2 \phi+\cos 2 x)^{-3 / 2} d x \\
& +z^{-\frac{1}{2}}(2-z)^{-\frac{1}{2}}(1-\cos 2 \phi)^{-\frac{1}{2}} .
\end{aligned}
$$

The main steps in the proof are the estimates of the two integral terms in (27) as $z \rightarrow 0$. This is done in the following two lemmas that we will prove afterwards.

Lemma 1. $\int_{0}^{\bar{x}^{\star}(z)}(z-\cos 2 \phi+\cos 2 x)^{-3 / 2} d x=O\left(z^{\frac{1}{2}}\right) \quad$ as $\quad z \rightarrow 0$.

Lemma 2. $\int_{0}^{\phi}(z-\cos 2 \phi+\cos 2 x)^{-3 / 2} d x=z^{-\frac{1}{2}}\left(\frac{1}{\sin 2 \phi}+o(1)\right) \quad$ as $\quad z \rightarrow 0$.

Using these two lemmas in (27) we can write, as $z \rightarrow 0$,

$$
\begin{aligned}
2 \frac{\partial T_{B}}{\partial z}(z, \phi) & =-z^{-\frac{1}{2}}\left(\frac{1}{\sin 2 \phi}+o(1)\right)+z^{-\frac{1}{2}} \frac{1}{\sqrt{2}}(1-\cos 2 \phi)^{-\frac{1}{2}}+O\left(z^{\frac{1}{2}}\right) \\
& =-z^{-\frac{1}{2}}\left(\frac{\sqrt{2}(1-\cos 2 \phi)^{\frac{1}{2}}-\sin 2 \phi}{\sqrt{2} \sin 2 \phi(1-\cos 2 \phi)^{\frac{1}{2}}}+o(1)\right) .
\end{aligned}
$$

Consider the function defined in the interval $[0, \pi)$ by $h(x)=2(1-\cos x)-\sin ^{2} x$. Clearly $h(0)=0$ and $h^{\prime}(x)>0$ for $x \in(0, \pi)$. Thus $h(x)>0$ if $x \in(0, \pi)$. This implies that $2(1-\cos 2 \phi)>\sin ^{2} 2 \phi$ for all $\phi \in\left(0, \frac{\pi}{2}\right)$ and the monotonicity of $\sqrt{\cdot}$ entails $\sqrt{2}(1-\cos 2 \phi)^{\frac{1}{2}}>\sin 2 \phi$. Using this in (28) completes the proof of Proposition 2.

Proof of Lemma 1. We first need to look at the behavior of $x^{\star}$ : a simple application of the following generalized Taylor expansion,

$$
\arccos (1-x)=\sqrt{2 x}+\frac{(2 x)^{\frac{3}{2}}}{24}+O\left(x^{2}\right) \quad \text { as } \quad x \rightarrow 0 .
$$

allows us to write

$$
\bar{x}^{\star}(z)=\frac{1}{\sqrt{2}} z^{\frac{1}{2}}+\frac{\sqrt{2}}{24} z^{\frac{3}{2}}+O\left(z^{2}\right), \quad \text { as } z \rightarrow 0 .
$$

Since $1-\cos 2 \phi=z-\cos 2 \phi+\cos 2 x^{\star}(z)<z-\cos 2 \phi+\cos 2 x<z+2$, and using (30), we get, as $z \rightarrow 0$,

$$
\frac{1}{4} z^{\frac{1}{2}}+O\left(z^{\frac{3}{2}}\right)<\int_{0}^{\bar{x}^{\star}(z)}(z-\cos 2 \phi+\cos 2 x)^{-3 / 2} d x \leqslant \frac{1}{\sqrt{2}}(1-\cos 2 \phi)^{-\frac{3}{2}} z^{\frac{1}{2}}+O\left(z^{\frac{3}{2}}\right),
$$

which proves Lemma 1. 
14 F.P. DA COSTA, M. GRINFELD, N. J. MOTTRAM, J.T. PINTO, AND K. XAYXANADASY

Proof of Lemma 2. Consider the trigonometric identity

$$
\cos a-\cos b=2 \sin \frac{b+a}{2} \sin \frac{b-a}{2}
$$

and use it to write the integral in the statement of the lemma as

$$
\int_{0}^{\phi}(z+2 \sin (\phi+x) \sin (\phi-x))^{-3 / 2} d x
$$

Considering the change of variable $x \mapsto t$ defined by $z t=\sin (\phi-x)$ we have $\frac{d t}{d x}=-\frac{1}{z} \sqrt{1-(z t)^{2}}$, and the integral becomes

$$
\begin{gathered}
z^{-\frac{1}{2}} \int_{0}^{\frac{\sin \phi}{z}} \frac{1}{\sqrt{1-(z t)^{2}}(1+2 t \sin (2 \phi-\arcsin (z t)))^{\frac{3}{2}}} d t \\
=z^{-\frac{1}{2}} \int_{0}^{+\infty} \underbrace{\frac{\mathbf{1}_{\left(0, \frac{\sin \phi}{z}\right)}(t)}{\sqrt{1-(z t)^{2}}(1+2 t \sin (2 \phi-\arcsin (z t)))^{\frac{3}{2}}}}_{=: f(z, t)} d t
\end{gathered}
$$

where $\mathbf{1}_{(a, b)}(t)$ is the characteristic function of the interval $(a, b)$, namely $\mathbf{1}_{(a, b)}(t)=$ 1 if $t \in(a, b)$ and zero otherwise. Observe now that

$$
\lim _{z \rightarrow 0} f(z, t)=\frac{1}{(1+2 t \sin 2 \phi)^{\frac{3}{2}}}=: f(t), \quad \text { pointwise in } t,
$$

and note also that, since $0 \leqslant z t \leqslant \sin \phi$ and $0<\phi<\frac{\pi}{2}$, we conclude that $\sin (2 \phi-$ $\arcsin (z t))$ is positive and bounded away from zero satisfying ${ }^{2} \sin (2 \phi-\arcsin (z t))>$ $\sin 2 \phi \wedge \sin \phi=: m(\phi)>0$. Also $\sqrt{1-(z t)^{2}} \geq \sqrt{1-\sin ^{2} \phi}=\cos \phi>0$. From these it follows that

$$
0<f(z, t) \leqslant \frac{1}{\cos \phi} \frac{\mathbf{1}_{\left(0, \frac{\sin \phi}{z}\right)}(t)}{(1+2 t m(\phi))^{\frac{3}{2}}} \leqslant \frac{1}{\cos \phi} \frac{1}{(1+2 t m(\phi))^{\frac{3}{2}}}=: \tilde{f}(t)
$$

and $\tilde{f}$ is integrable in $[0,+\infty)$. Hence, by the Lebesgue's dominated convergence theorem applied to the integral in (32) we conclude that, as $z \rightarrow 0$,

$$
\int_{0}^{+\infty} f(z, t) d t=\int_{0}^{+\infty} \frac{1}{(1+2 t \sin 2 \phi)^{\frac{3}{2}}} d t+o(1)=\frac{1}{\sin 2 \phi}+o(1)
$$

and this concludes the proof of Lemma 2 .

The next proposition is an elementary result in real analysis that we state and prove for completeness.

Proposition 3. Let $\psi:[0, c) \rightarrow \mathbb{R}$ be continuous in $[0, c)$, differentiable in $(0, c)$, and assume $\psi^{\prime}(x) \rightarrow-\infty$ as $x \rightarrow 0$. Then, the following holds true: $\psi^{\prime}(0):=$ $\lim _{\xi \rightarrow 0^{+}} \frac{\psi(\xi)-\psi(0)}{\xi}=-\infty$.

\footnotetext{
${ }^{2}$ Recall the notation $a \wedge b=\min \{a, b\}$,
} 
Proof. Fix $\xi \in(0, c)$ and apply the mean value theorem to the interval $[0, \xi]$. We conclude that there exists $x_{\xi} \in(0, \xi)$ such that

$$
\frac{\psi(\xi)-\psi(0)}{\xi}=\psi^{\prime}\left(x_{\xi}\right) \text {. }
$$

Passing to the limit as $\xi \rightarrow 0$ in both sides of this expression, and using the assumption about $\psi^{\prime}(x)$ when $x$ approaches 0 in the right-hand side, we conclude the proof.

We can now apply Propositions 2 and 3 to immediately conclude that

Proposition 4. $\frac{\partial T_{B}}{\partial z}(0, \phi)=-\infty$

The results of Propositions 2 and 4 imply that the time taken by a solution of type $B$ close to the bifurcation point is smaller than the time $T^{*}$ taken by the critical solution $\gamma^{*}$.

4.4. On the global behavior of type $B$ solutions branch. The result obtained in the previous section for the time taken by a type $B$ solution is of a local character: it is valid when the type $B$ solution is close to the critical one $\gamma^{*}$, i.e., when the value of the parameter indexing the solution (be it $\alpha, \beta, y(-L)$, or $z$ ) is sufficiently close to the value of the corresponding one in the critical one $\left(\phi, \phi^{\star}, 0\right.$ or 0 , respectively).

From the study presented in section 3, we concluded that the type $B$ branch of solutions can be continued away from the neighborhood of the critical solution, and solutions on this branch, parameterized by the value of $z:=y(-L)^{2}$, only cease to exist when $z>2$. To understand the global behavior of this branch for $z \in(0,2)$ we need to know the behavior of the map $z \mapsto T_{B}(z)$. In particular, if we could prove that this function is convex, we could conclude that the branch of type $B$ solutions has a unique saddle-node bifurcation point.

Differentiating (27) with respect to $z$ we get, after some algebraic manipulations,

$$
\begin{aligned}
4 \frac{\partial^{2} T_{B}}{\partial z^{2}}(z, \phi)= & 3 \int_{0}^{\phi}(z-\cos 2 \phi+\cos 2 x)^{-5 / 2} d x \\
& +3 \int_{0}^{\bar{x}^{\star}(z)}(z-\cos 2 \phi+\cos 2 x)^{-5 / 2} d x \\
& +z^{-\frac{3}{2}}(2-z)^{-\frac{3}{2}}(1-\cos 2 \phi)^{-\frac{3}{2}} g(z, \phi),
\end{aligned}
$$

where

$$
g(z, \phi):=z^{2}-(2 \cos 2 \phi) z-2(1-\cos 2 \phi) .
$$

When $g$ is negative, the sign of $\frac{\partial^{2} T_{B}}{\partial z^{2}}(z, \phi)$ depends on the balance between the two positive integrals and the (negative) last term in (33), and its determination seems to be a challenging problem. However, close to the border $z=0$ we can compute the sign of $\frac{\partial^{2} T_{B}}{\partial z^{2}}$ using the asymptotic technique employed in the proof of Lemma 2. The result is stated in the next lemma.

Proposition 5. $\frac{\partial^{2} T_{B}}{\partial z^{2}}(z, \phi)>0$ as $z \rightarrow 0$. 
Proof. From (33) we have

$4 \frac{\partial^{2} T_{B}}{\partial z^{2}}(z, \phi) \geqslant 3 \int_{0}^{\phi}(z-\cos 2 \phi+\cos 2 x)^{-5 / 2} d x+z^{-\frac{3}{2}}(2-z)^{-\frac{3}{2}}(1-\cos 2 \phi)^{-\frac{3}{2}} g(z, \phi)$.

Using the trigonometric identity (31) and the change of variable $x \mapsto t$, with $z t=$ $\sin (\phi-x)$, in the integral in (35), we can write

$$
\int_{0}^{\phi}(z-\cos 2 \phi+\cos 2 x)^{-\frac{5}{2}} d x=z^{-\frac{3}{2}} \int_{0}^{+\infty} \frac{\mathbf{1}_{\left(0, \frac{\sin \phi}{z}\right)}(t)}{\sqrt{1-(z t)^{2}}(1+2 t \sin (2 \phi-\arcsin (z t)))^{\frac{5}{2}}} d t .
$$

Observing that the integral in right-hand side is like (32) with $-1 / 2$ changed to $-3 / 2$ and $3 / 2$ to $5 / 2$, we can apply the argument in the proof of Lemma 2 to obtain, as $z \rightarrow 0$,

$\int_{0}^{\phi}(z-\cos 2 \phi+\cos 2 x)^{-\frac{5}{2}} d x=z^{-\frac{3}{2}} \int_{0}^{+\infty} \frac{1}{(1+2 t \sin 2 \phi)^{\frac{5}{2}}} d t+o(1)=\frac{1}{3 \sin 2 \phi}+o(1)$.

Now, using the definition of $g,(34)$, to write $g(z, \phi)=-2(1-\cos 2 \phi)+O(z)$ as $z \rightarrow 0$, observing that $\sin 2 \phi=\left(1-\cos ^{2} 2 \phi\right)^{\frac{1}{2}}=(1-\cos 2 \phi)^{\frac{1}{2}}(1+\cos 2 \phi)^{\frac{1}{2}}$, and substituting (36) into (35), we obtain the following, as $z \rightarrow 0$,

$$
4 \frac{\partial^{2} T_{B}}{\partial z^{2}}(z, \phi) \geqslant z^{-\frac{3}{2}} \frac{1}{(1-\cos 2 \phi)^{\frac{1}{2}}}\left(\frac{1}{(1+\cos 2 \phi)^{\frac{1}{2}}}-\frac{1}{\sqrt{2}}+o(1)\right)>0
$$

where the positivity is due to $1+\cos 2 \phi \in(0,2)$, and hence $(1+\cos 2 \phi)^{-\frac{1}{2}}>\frac{1}{\sqrt{2}}$.

Numerical computations using the software Mathematica ${ }^{\circledR}$ provide very convincing evidence for the convexity of $T_{B}(\cdot, \phi)$ everywhere in the rectangle $(0,2) \times\left(0, \frac{\pi}{2}\right)$. Unfortunately, despite repeated efforts using various approaches, some described in the Appendix, we were not yet able to establish this result rigorously.

Thus, we state the following

Conjecture 1. For each fixed $\phi \in\left(0, \frac{\pi}{2}\right)$, the function $z \mapsto T_{B}(z, \phi)$ is convex.

For the remainder of this paper we assume this conjecture to hold. We reformulate it as a statement about $B$ type solutions.

Claim 1. The branch of solutions $B$ has a unique saddle-node bifurcation point.

4.5. Behavior of the type $A$ solution branch. From (20) we know that solutions of type $A$ satisfy $T_{A}(\alpha)=2 T(\alpha)-T_{B}(\alpha)$. By the results of section 4.3 , we know that $T_{B}$ is decreasing when $\alpha>\phi$ close to $\phi$, and, by Proposition $1, T$ is always increasing. Thus, we conclude that the time $T_{A}$ taken by solutions of type $A$ close to the bifurcation point is larger than the time $T^{*}$ taken by the critical solution $\gamma^{*}$.

By putting together all previous (analytical and numerical) results we conclude that the bifurcation diagram of (14)-(15) about $T^{*}$ is the one shown qualitatively in Figure 5. 


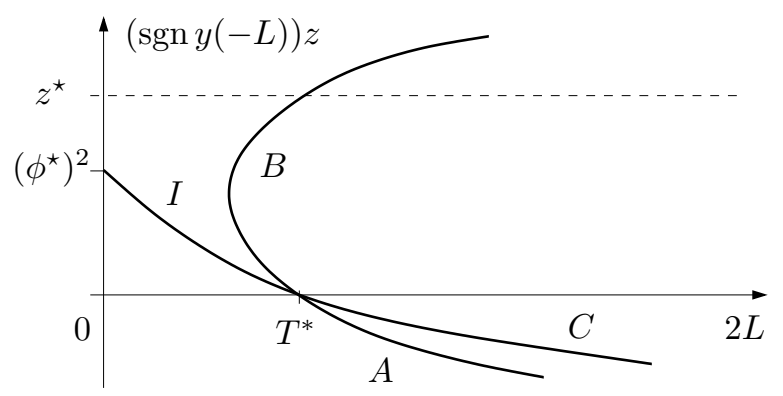

Figure 5. Qualitative sketch of the local bifurcation around $T^{*}:=T(\phi)$, and global behaviour of the bifurcating branches with $T<T^{*}$ assuming the behaviour of type $B$ solutions stated in Conjecture 1. The plotted case corresponds to $\phi \in\left(0, \frac{\pi}{4}\right)$. When $\phi \in\left(\frac{\pi}{4}, \frac{\pi}{2}\right)$ the end point of the branch $I$ occurs above the homoclinic orbit, and thus $\left(\phi^{\star}\right)^{2}>z^{\star}:=1+\cos 2 \phi$.

\section{OTHER BIFURCATIONS}

As was the case of system (14) with non-homogeneous Dirichlet boundary conditions studied in $[3,4]$, in the present system (14)-(15) with large values of $L$ we can have solutions turning several times around the origin in the region of the cylindrical phase plane bounded by the two homoclinic orbits.

To study these cases we use the same principle of perturbing critical solutions $\gamma_{k}^{*}$ defined as $\gamma^{*}$ (i.e., satisfying, in addition to (15), homogeneous boundary conditions $y(-L)=0$ and $x(L)=0$ ) but now turning $k$ times around the origin. We have also four distinct types of solutions that we can denote by $I_{k}, A_{k}, B_{k}$ and $C_{k}$, analogous to $I, A, B$ and $C$, which can be considered to be the cases with $k=0$ (i.e., orbits that do not have any complete turn around the origin). The time spent by these solutions with $z$ sufficiently close to zero is obtained by adding $4 k T(\alpha)$ to the times spent by the corresponding $k=0$ solutions, e.g.

$$
T_{I_{k}}(z, \phi)=4 k T(z)+T_{I_{0}}(z, \phi),
$$

and likewise for the other types of orbits.

To build a global picture of the $I_{k}$ solution branch as $z$ increases away from $z=0$ we need to start by recalling what happens with the $I$ branch (i.e.: with the case $k=0$ ). This was studied in sections 3 and 4.1, and illustrated in figures 3 and 5 : the $I$ branch of solutions collapses to a single point and disappears when $z=\sqrt{\phi^{\star}}$.

In the case of $I_{k}$ with $k \geqslant 1$ an entirely different behaviour takes place: the $I_{k}$ branch of solutions exists for all $z<z^{\star}:=1+\cos 2 \phi$, where $z^{\star}$ is the value of $z$ of the point on the homoclinic orbit with $x=-\phi$, and $T_{I_{k}}(z) \rightarrow+\infty$ when $z \rightarrow z^{\star}$. In fact we have the two situations we now describe:

Suppose $\phi \in\left(\frac{\pi}{4}, \frac{\pi}{2}\right)$, then $\cos 2 \phi<0$ and thus $z^{\star}=1+\cos 2 \phi<1-\cos 2 \phi=\left(\phi^{\star}\right)^{2}$ which implies that the point where the branch of solutions of type $I$ collapse, $P^{\star}=$ $\left(-\phi, \phi^{\star}\right)$ in Figure 3, is above the point $\left(-\phi, z^{\star}\right)$ on the homoclinic. This implies that, as $z \uparrow z^{\star}$, the solutions of type $I_{k}$ converge to the homoclinic net constituted 
by the point $\left(-\frac{\pi}{2}, 0\right) \equiv\left(\frac{\pi}{2}, 0\right)$ and the two homoclinic orbits, as illustrated in Figure 6 . The time spent will, obviously, go to $+\infty$.

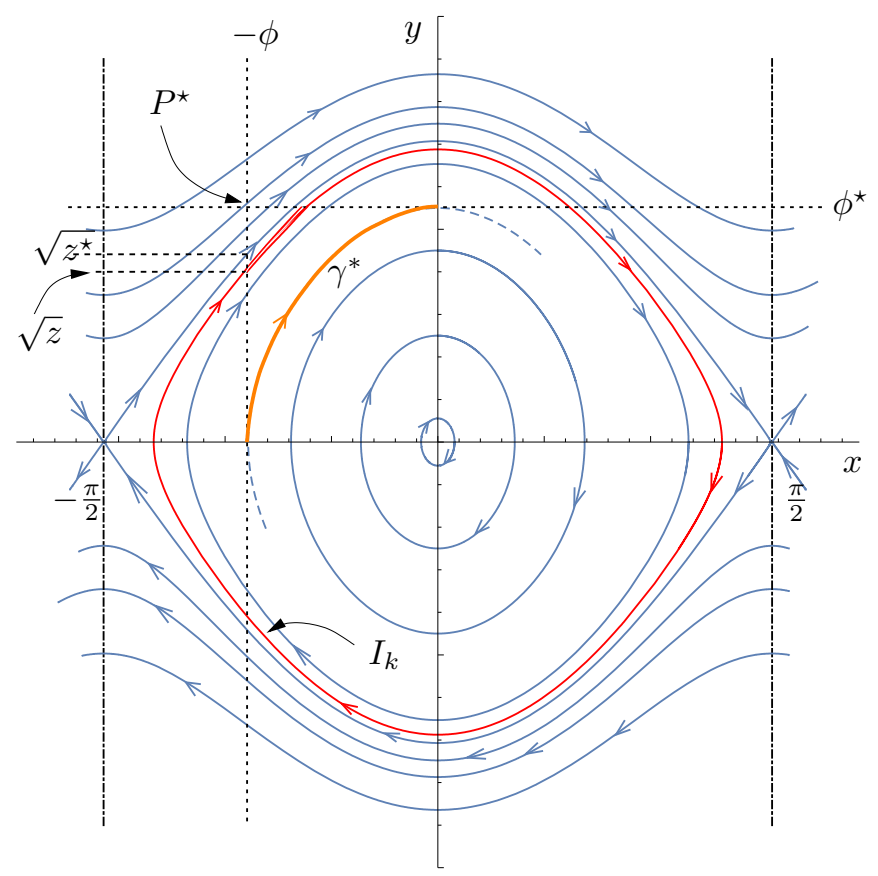

FiguRE 6 . An orbit of type $I_{k}$, with $k=1$, and with $z$ close to $z^{\star}$. The case shown corresponds to $\phi \in\left(\frac{\pi}{4}, \frac{\pi}{2}\right)$, in which case $P^{\star}$ is outside the homoclinic net.

Assume now $\phi \in\left(0, \frac{\pi}{4}\right)$. Then $\cos 2 \phi>0$ and thus $z^{\star}=1+\cos 2 \phi>1-\cos 2 \phi=$ $\left(\phi^{\star}\right)^{2}$ which implies that the point $P^{\star}$ is now located inside the homoclinic net, as illustrated in Figure 7. It is clear from this figure that the time spent by these solutions of type $I_{k}$ is given by

$$
T_{I_{k}}(z, \phi)=4 k T(z)-T_{I_{0}}(z, \phi) .
$$

From Proposition 1, section 4.1, and from $V(-\alpha, 0)=V(-\phi, \sqrt{z})$, which results in the relation $\alpha=\frac{1}{2} \arccos (\cos 2 \phi-z)$, it is easy to conclude that solutions whose time map is given by (39) satisfy

$$
\frac{\partial T_{I_{k}}}{\partial z}(z, \phi)>0
$$

and hence the corresponding solution branch in the bifurcation diagram is the graph of a monotonic increasing function converging to the horizontal asymptote $z=z^{\star}$ as the time $L$ converges to $+\infty$.

On the other hand, for $I_{k}$ solutions that do not enclose the point $P^{\star}$ (i.e., those illustrated in Figure 6), the computation of $\frac{\partial T_{I_{k}}}{\partial z}(z, \phi)$ faces exactly the same problems as we confronted in section 4.4 for the global behaviour of solution branch $B$. 


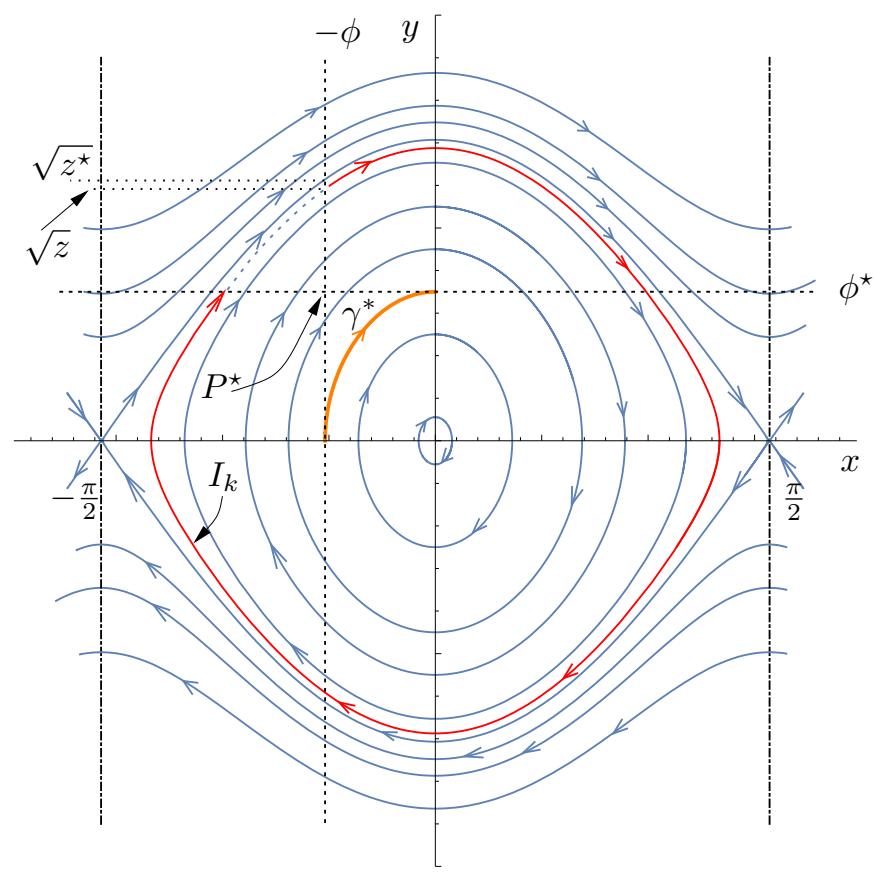

Figure 7 . An orbit of type $I_{k}$, with $k=1$, and with $z$ close to $z^{\star}$. The case shown corresponds to $\phi \in\left(0, \frac{\pi}{4}\right)$, in which case $P^{\star}$ is inside the homoclinic net.

We can repeat the arguments in section 4.3 to conclude that $\frac{\partial T_{I_{k}}}{\partial z}(z, \phi) \rightarrow-\infty$ as $z \rightarrow 0$. Since, as we noted above, $T_{I_{k}}(z, \phi) \rightarrow+\infty$ as $z \rightarrow z^{\star}$, we conclude that the $T_{I_{k}}(z, \phi)$ must have at least one minimum for some ${ }^{3} \sqrt{z} \in\left(0, \phi^{\star}\right)$. A numerical study entirely analogous the one presented in the Appendix allows us to believe that Claim 1 is also valid for the branches $I_{k}$ with $k \geqslant 1$.

From the fact that the time maps for the branches $B_{k}$ with $k \geqslant 1$ are, like in (38), obtained from the one of branch $B$ by adding $4 k T(z)$, the conclusion we reached for the branches $I_{k}$ is repeated for the $B_{k}$ s.

Thus, from the discussion above and assuming Claim 1 holds true for the branches $I_{k}$ and $B_{k}$ with $k \geqslant 1$, the bifurcation scheme for the solution branches with $k \geqslant 1$ is shown qualitatively in Figure 8.

In addition to these solutions, there are two other families of solutions, which we denote by $D_{k}$ and $D_{k}^{\prime}$. These correspond to solutions analogous to $B$ and $B^{\prime}$ but are located above the homoclinic orbit with positive $y$-component, and turn around the cylindrical phase space $k$ times (see example in Figure 9).

\footnotetext{
${ }^{3}$ Observe that, due to $(40)$, if the interval $\left(\phi^{\star}, \sqrt{z^{\star}}\right)$ is not empty the piece of the solution branch $I_{k}$ with $\sqrt{z}$ in this interval is monotonic and so the minima of the $I_{k}$ branch must necessarily correspond to values $\sqrt{z}<\phi^{\star}$.
} 


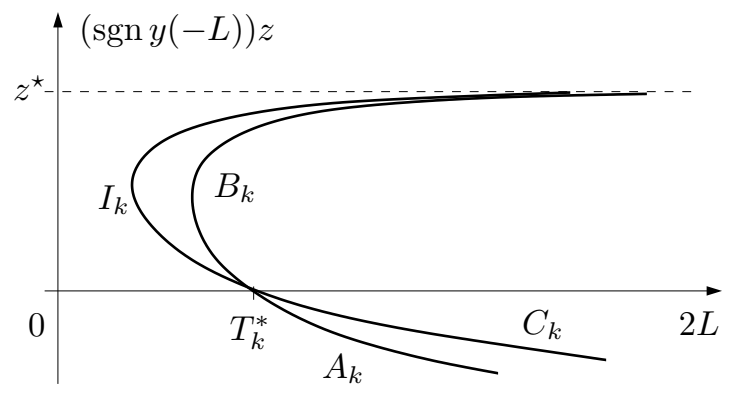

Figure 8. Qualitative sketch of the bifurcating branches $I_{k}, A_{k}, B_{k}$ and $C_{k}$, with $k \geqslant 1$, assuming the behaviour of the branch $B_{0}$ stated in Claim 1 is also valid for branches $I_{k}$ and $B_{k}$. Here $T_{k}^{*}:=4 k T(\phi)$.

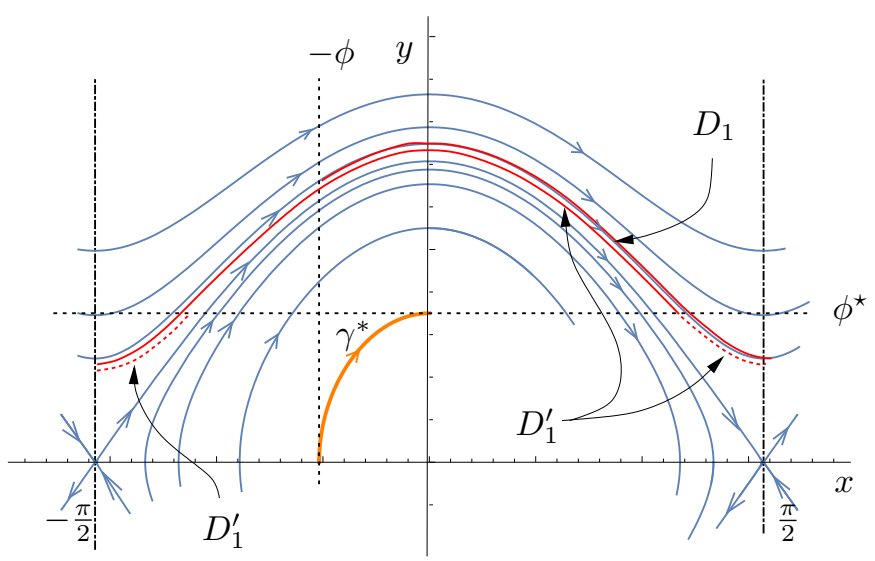

Figure 9 . Orbits of type $D_{k}$ and $D_{k}^{\prime}$, with $k=1$. The orbit $D_{1}^{\prime}$ is represented by the complete line (dashed and undashed); $D_{1}$ is represented by the undashed part only. In this plot each time the orbit $D_{1}$ or $D_{1}^{\prime}$ passes through a same point the line is slightly deflected in order to facilitate the reading.

The amount of time taken by these solutions to travel between one boundary condition and the other are given by

$$
\begin{aligned}
& T_{D_{k}}(z, \phi):=2 k T_{1}\left(z, \frac{\pi}{2}\right)+T_{1}(z, \phi)+T_{1}\left(z, \bar{x}^{\star}(z)\right) \\
& T_{D_{k}^{\prime}}(z, \phi):=2(k+1) T_{1}\left(z, \frac{\pi}{2}\right)+T_{1}(z, \phi)-T_{1}\left(z, \bar{x}^{\star}(z)\right),
\end{aligned}
$$

where, for each $\phi \in\left(0, \frac{\pi}{2}\right), z$ varies from $z^{\star}:=1+\cos 2 \phi$, the value of ordinate square of the point of intersection of $x=-\phi$ and the homoclinic orbit, obtained from $V\left(-\frac{\pi}{2}, 0\right)=V\left(-\phi, \sqrt{z^{\star}}\right)$, and 2, the biggest value of $z$ for which the solution intersects the line $y=\phi^{\star}$.

We can infer directly from Figure 9 that when $z=2$ the end points of $D_{k}$ and $D_{k}^{\prime}$ are the same (and coincide with the point $\left(\frac{\pi}{2}, \phi^{\star}\right)$.) This can also be obtained 
from (41)-(42):

$$
\begin{aligned}
T_{D_{k}^{\prime}}(z, \phi) & =2(k+1) T_{1}\left(z, \frac{\pi}{2}\right)+T_{1}(z, \phi)-T_{1}\left(z, \bar{x}^{\star}(z)\right) \\
& =T_{D_{k}}(z, \phi)+2\left(T_{1}\left(z, \frac{\pi}{2}\right)-T_{1}\left(z, \bar{x}^{\star}(z)\right)\right) \\
& \geqslant T_{D_{k}}(z, \phi),
\end{aligned}
$$

since, from its definition, $T_{1}(z, \cdot)$ is monotonic increasing, and, by $(24), \bar{x}^{\star}(z) \leqslant \frac{\pi}{2}$ with equality only when $z=2$, because $\bar{x}^{\star}(2)=\frac{1}{2} \arccos (-1)=\frac{\pi}{2}$.

From (43) we conclude that, for each $k$, the branches $D_{k}$ and $D_{k}^{\prime}$ exist for all $z \in\left(z^{\star}, 2\right)$ and, in a bifurcation diagram with time as the bifurcation variable and $z$ as the dependent variable, the $D_{k}$ branch will always be to the left of the $D_{k}^{\prime}$, except at one single point, with ordinate $z=2$, where they coincide.

To study the monotonicity of the branches $D_{k}$ and $D_{k}^{\prime}$ we again use the time maps (41) and (42), respectively. The last is easier: differentiating (42) with respect to $z$ we obtain, after some algebraic manipulations,

$$
\begin{aligned}
\frac{\partial T_{D_{k}^{\prime}}}{\partial z}(z, \phi)= & -\left(k+\frac{1}{2}\right) \int_{0}^{\frac{\pi}{2}}(z-\cos 2 \phi+\cos 2 x)^{-3 / 2} d x \\
& -\frac{1}{2} \int_{0}^{\phi}(z-\cos 2 \phi+\cos 2 x)^{-3 / 2} d x \\
& -\frac{1}{2} \int_{\bar{x}^{\star}(z)}^{\frac{\pi}{2}}(z-\cos 2 \phi+\cos 2 x)^{-3 / 2} d x \\
& -\frac{1}{2} z^{-\frac{1}{2}}(2-z)^{-\frac{1}{2}}(1-\cos 2 \phi)^{-\frac{1}{2}} \\
< & 0
\end{aligned}
$$

and $\frac{\partial T_{D_{k}^{\prime}}}{\partial z} \rightarrow-\infty$ when $z \rightarrow z^{\star}$ and when $z \rightarrow 2$.

The same computation for the branch $D_{k}$ runs into the difficulties already encountered before when studying the branches $B_{k}$ (with $\left.k \geqslant 0\right)$ and $I_{k}$ (with $k \geqslant 1$ ). The expression for the derivative of the time map is

$$
\begin{aligned}
\frac{\partial T_{D_{k}}}{\partial z}(z, \phi)= & -(k+1) \int_{0}^{\frac{\pi}{2}}(z-\cos 2 \phi+\cos 2 x)^{-3 / 2} d x \\
& -\frac{1}{2} \int_{0}^{\phi}(z-\cos 2 \phi+\cos 2 x)^{-3 / 2} d x \\
& -\frac{1}{2} \int_{0}^{\bar{x}^{\star}(z)}(z-\cos 2 \phi+\cos 2 x)^{-3 / 2} d x \\
& +\frac{1}{2} z^{-\frac{1}{2}}(2-z)^{-\frac{1}{2}}(1-\cos 2 \phi)^{-\frac{1}{2}},
\end{aligned}
$$

and $\frac{\partial T_{D_{k}}}{\partial z} \rightarrow-\infty$ when $z \rightarrow z^{\star}$, and $\frac{\partial T_{D_{k}}}{\partial z} \rightarrow+\infty$ when $z \rightarrow 2$. The existence of a unique minimum of $T_{D_{k}}(\cdot, \phi)$ can be checked numerically like was done in the case of the $B$ branch in the Appendix, but, as there, the rigorous proof eludes us at present. All numerical evidence points to the validity of Conjecture 1 also for the function $z \mapsto T_{D_{k}}(z, \phi)$, and assuming this, we have the equivalent of Claim 1, so that the situation with branches $D_{k}$ and $D_{k}^{\prime}$ is as illustrated qualitatively in Figure 10 . 


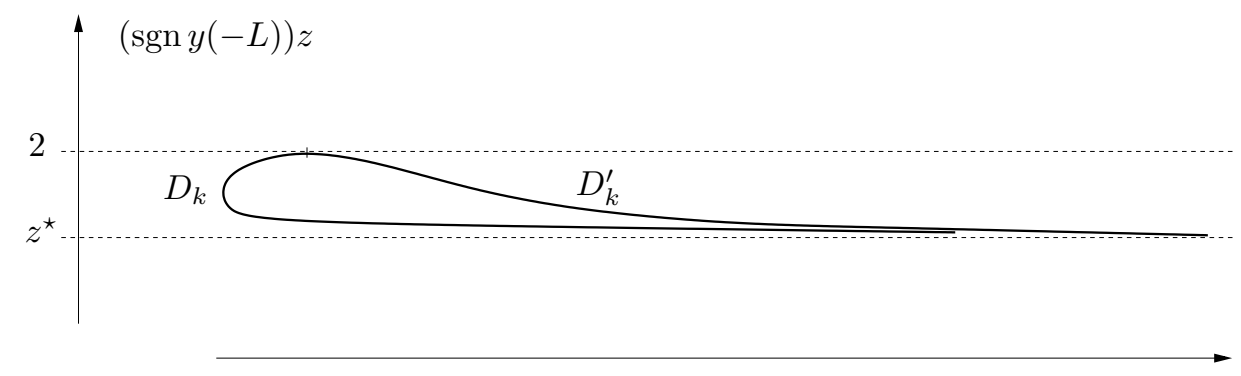

FIGURE 10. Qualitative sketch of solution branches $D_{k}$ and $D_{k}^{\prime}$, with $k \geqslant 1$, assuming that the behaviour of branch $B$ stated in Conjecture 1 is also valid for branches $D_{k}$.

\section{Discussion}

Gathering the results and discussions from the previous sections we can construct the qualitative sketch of the bifurcation diagram for solutions of (14)-(15) in Figure 11.

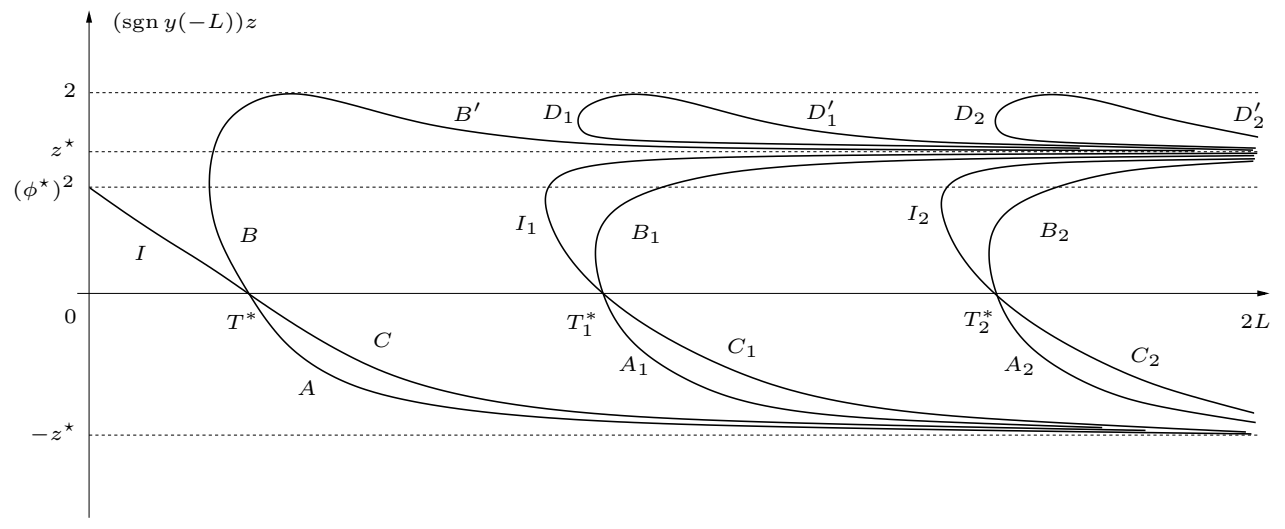

FiguRE 11. Qualitative sketch of the bifurcation diagram for system (14)-(15) with $\phi \in\left(0, \frac{\pi}{4}\right)$, assuming validity of Conjecture 1 for the relevant branches, as explained in the text. For the notation used in this figure see the text.

We can consider the physical implications of these results by considering experimental situations in which we increase the parameter $L$ from zero, or equivalently, through (7), increasing the liquid crystal layer thickness $d$, while keeping all other parameters fixed. As $L \rightarrow 0(d \rightarrow 0)$ we see from Figure 11, branch $I$, that $x^{\prime}(-L) \rightarrow \phi^{\star}$, or in dimensional terms, $d \varphi / d x_{3} \rightarrow q$ at $x_{3}=0$. Indeed, in this limit $d \varphi / d x_{3} \rightarrow q$ throughout the layer and the solution is in fact $\varphi\left(x_{3}\right)=q x_{3}-\varphi_{0}$. This behaviour is to be expected, since for a very thin layer the boundary conditions and internal elasticity effects dominate the orienting field and we recover the solution of the system for $F=0$. 
As $L$ increases, the field effect can reorient the director in the layer and this leads to a reduction in $x^{\prime}(-L)$ until a fold bifurcation (branch $B$ emerges) and the transcritical bifurcation at $2 L=T^{*}$ occurs. In dimensional variables this bifurcation occurs at $d=d^{*}=T\left(\varphi_{0}\right) \sqrt{\left(2 K_{2}\right) /\left(\chi F^{2}\right)}$. The emergence of other solution branches as $d$ increase is to be expected, and similar effects have been seen in many other papers, cf. $[2,12,13,16,20,24]$. The presence of multiple solutions is due to the ability of the orienting field to unwind the chiral helix, as mentioned in subsection 1.2. At low field strengths there are a fixed number of director rotations that form the chiral helix within the liquid crystal layer, as field strength increases and dominates elastic effects, the number of director rotations may reduce. The form of $d^{*}$ and the related emergence of other solution branches is therefore to be expected: the critical layer thickness is larger for increasing elastic effects, $K_{2}$, and for decreasing field effects, $\chi F^{2}$. It should also be noted that two properties of $T\left(\varphi_{0}\right)$, namely that $T(0)=\pi /(2 \sqrt{2})$ and $T\left(\varphi_{0}\right) \rightarrow \infty$ as $\varphi_{0} \rightarrow \pi / 2$, provide interesting results. The first property means that when $\varphi_{0}=0$, so that the director orientation at $x_{3}=0$ is perpendicular to the orienting field, the critical layer thickness $d^{*}$ is a minimum, and equal to

$$
d^{*}=\frac{\pi}{2 F} \sqrt{\frac{K_{2}}{\chi}},
$$

which is half the critical thickness for the classical transition, and is to be expected in a layer with one Dirichlet and one Neumann boundary condition. The second property of $T\left(\varphi_{0}\right)$ indicates that the critical thickness $d^{*}$ goes to infinity as $\varphi_{0} \rightarrow$ $\pi / 2$, in other words when the director at the lower surface is aligned with the field direction. Again this is to be expected since alignment between the director at $x_{3}=0$ and the applied field will stabilise the primary $I$ solution branch for larger layer thicknesses, moving the bifurcation to larger values of $L$ (or $d$ ).

The presence of further bifurcations, and therefore additional solution branches, as $L$ (or $d$ ) increases, is also to be expected and has been predicted as unwinding transitions in previous work (for instance [16].) For a thicker liquid crystal layer, and a fixed chirality $q$, more rotations of the director may be contained within the layer, and there are are more possible unwindings of the helix. As $d$ increases, and each solution branch of type $I$ appears, the number of possible solutions increases because additional rotations of the director can now be contained within the layer.

On the other hand, the presence of the $D_{k}$ and $D_{k}^{\prime}$ solution branches is intriguing and not immediately explained in terms of a balance of elastic, field and surface effects. However, a numerical investigation (see below in Figure 13) shows that the solutions on these branches consist of regions where the director aligns with the orienting field, so that $x \approx(2 n+1) \pi / 2(n \in \mathbb{Z})$ separated by regions where the natural chirality dominates, so that $y \approx \phi^{\star}$. We find that there are two regions aligned with the field on solution branch $D_{1}$, three regions aligned with the field on solution branch $D_{2}$ etc. Further investigation of these solution branches, as well as the parameter dependence of the point at which the $D_{k}$ branches folds, would be an interesting topic for future work. In the context of the phase plane described in previous sections we see that the unwinding transitions, i.e. changes in pitch and jumps between branches associated with the original work of for instance de Gennes 
[5] and Dreher [8], are associated with solutions transitioning between being inside to outside the heteroclinic orbits.

Observe that the boundary value problem (12)-(13) that we have been studying in this paper is the steady state problem of models describing the time evolution of the director field. In the absence of flow of the liquid crystal the simplest such model is the gradient flow of the free energy $W$, which is equivalent to the so-called low-Ericksen number limit of the full fluid dynamics of the system. In dimensionless form and with the notation used before (with $\tau$ the non-dimensional physical time), the initial-boundary value problem governing the dynamic behaviour of the director field is then

$$
\left\{\begin{array}{l}
\frac{\partial x}{\partial \tau}(\tau, t)=\frac{\partial^{2} x}{\partial t^{2}}(\tau, t)+\sin 2 x(\tau, t), \quad(\tau, t) \in \mathbb{R}^{+} \times(-L, L) \\
x(\tau,-L)=\phi, \quad \frac{\partial x}{\partial t}(\tau, L)=\phi^{\star}, \quad \tau \geqslant 0 \\
x(0, t)=\psi(t), \quad t \in[-L, L]
\end{array}\right.
$$

In this context a natural problem is the determination of the stability of the solution branches investigated in this paper when seen as steady-state solutions of (46). This is a problem requiring (and deserving) future work, but some preliminary numerical simulations suggest that branches $I, A, B^{\prime}, D_{1}^{\prime}$ and $D_{2}^{\prime}$ are made up of locally asymptotically stable equilibrium solutions, as well as the parts of branches $B, D_{1}$ and $D_{2}$ between the saddle-node point and the point connecting them with $B^{\prime}, D_{1}^{\prime}$ and $D_{2}^{\prime}$, respectively. One such simulation is presented in Figure 12 and shows the (numerically asymptotically stable) limit values to which numerical solutions of (46) with various initial conditions converge to when $\tau$ is large. For none of the initial conditions used in the numerical simulations did the system converge to a solution that corresponds to any of the branches $I_{k}$, and $A_{k}$, and to $B_{k}$ and $D_{k}$ below the saddle-node points. Though, as discussed above, further solution branches are to be expected as $d$ is increased, an intriguing finding of this paper is the numerical stability of the $D_{k}^{\prime}$ and part of the $D_{k}$ branches.

In Figure 13 convergence to solutions from each of the numerically stable branches in Figure 12 are shown. In Figure 13(a) we see that for the smallest value of the layer thickness, $2 L=0.01$, the director twist angle is almost constant and prescribed by the angle at the boundary $t=0$, where the Dirichlet condition is applied. On branch $I$, as $L$ increases we see that the increased layer thickness allows chirality to play a more important role and there is increased twist. On branches $A$ and $B$ (Figure 13(b) and (c)), which occur for larger values of the layer thickness $L$ than branch $I$, the orienting field affects the director orientation in the bulk of the layer, aligning the director to $x \approx-\pi / 2$ (branch $A$ ) and $x \approx \pi / 2$ (branch $B$ ). For even larger layer thicknesses, the solutions on branches $D_{1}, D_{1}^{\prime}$ and $D_{2}, D_{2}^{\prime}$ (Figure 13(d) and (e)) show field alignment at director angles $x \approx \pi / 2,3 \pi / 2$ and $x \approx \pi / 2,3 \pi / 2,5 \pi / 2$ respectively. Although, for the same layer thickness, branches $D_{1}, D_{1}^{\prime}$ and $D_{2}, D_{2}^{\prime}$ exhibit much larger director distortion, and therefore elastic energy, compared to branches $A$ and $B$, the numerical solution of the problem shows that they are locally asymptotically stable. Because of their high energy it is expected that, if they are indeed asymptotically stable, their basins of attraction will be small and thus these solutions may be difficult to create in an experimental setting, the system preferring to attain one of the lower energy solutions on branches $A$ or $B$ which are expected to have larger attraction basins. 


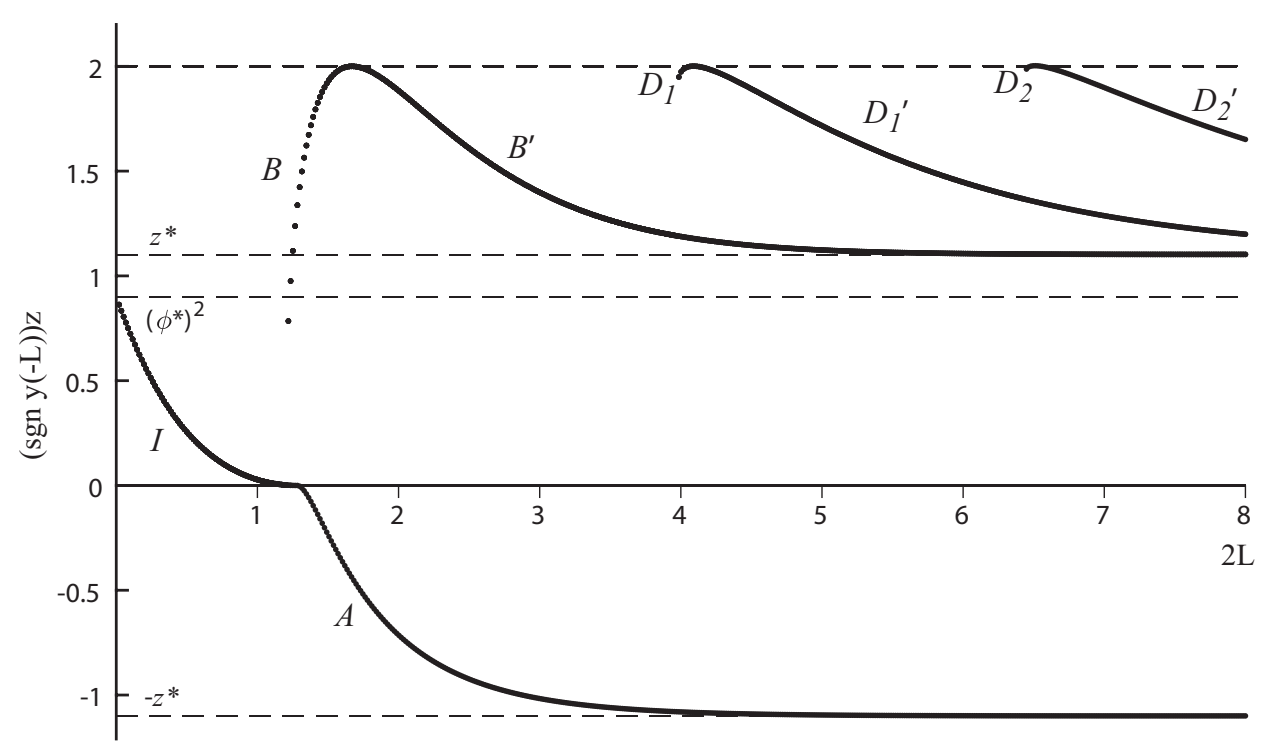

FiguRE 12. Numerically computed locally asymptotically stable equilibrium solution branches of (46) with $\phi=0.9$ and $\phi^{\star}=$ $\sqrt{1-\cos 2 \phi}$. The plot shows the value of $(\operatorname{sgn} y(-L)) z$ at large $\tau$, for varying values of $2 L$ and for various initial conditions, indicating that branches $I, A, B^{\prime}, D_{1}^{\prime}$ and $D_{2}^{\prime}$, as well as the parts of branches $B, D_{1}$ and $D_{2}$ between the saddle-node point and the point connecting them with $B^{\prime}, D_{1}^{\prime}$ and $D_{2}^{\prime}$, respectively consist of stable solutions. This figure should be compared with Figure 11.

To conclude, we have formulated in (4)-(6) a general boundary value problem for the steady state director configuration in a cholesteric liquid crystal subjected to a magnetic field. In (12)-(13) we have used a particular form of one of the boundary conditions for which we gave an almost complete description of the stationary solutions of the system. A complete study of (4)-(6), including its dynamic version (46), we leave for future analysis. As mentioned in Section 1.2, the second boundary condition in (15), with the condition $\phi^{\star}=\sqrt{1-\cos 2 \phi}$, although experimentally achievable, is rather restrictive. However, this restriction allows us to reduce the parameter space and to make the system more manageable in terms of the analysis and understanding. As referred to in the footnote in Section 1.2, preliminary results obtained in [15], using an approach similar to the one in the present paper, show that the transcritical bifurcation point at $T^{*}$ in Figure 11 is broken in the same way as in the twist nematic case when the antisymmetric strong boundary condition was changed to asymmetric (compare Figure 9 in [3], for the first case, with Figures 9 and 13 in [4] for the second). However, this analysis also indicates that when the field strength is close to the value determined by the condition $\phi^{\star}$ used in this paper, the two ratios of the three lengthscales in the system, $\phi_{0}^{*}$ and $L$ will be only slightly perturbed and therefore, although the bifurcation diagram is qualitatively different, in practical terms the difference may be very hard to identify, either numerically or experimentally. Clearly this is expected to change if the boundary value at $t=L$ is significanly different from the value considered in this 
26 F.P. DA COSTA, M. GRINFELD, N. J. MOTTRAM, J.T. PINTO, AND K. XAYXANADASY
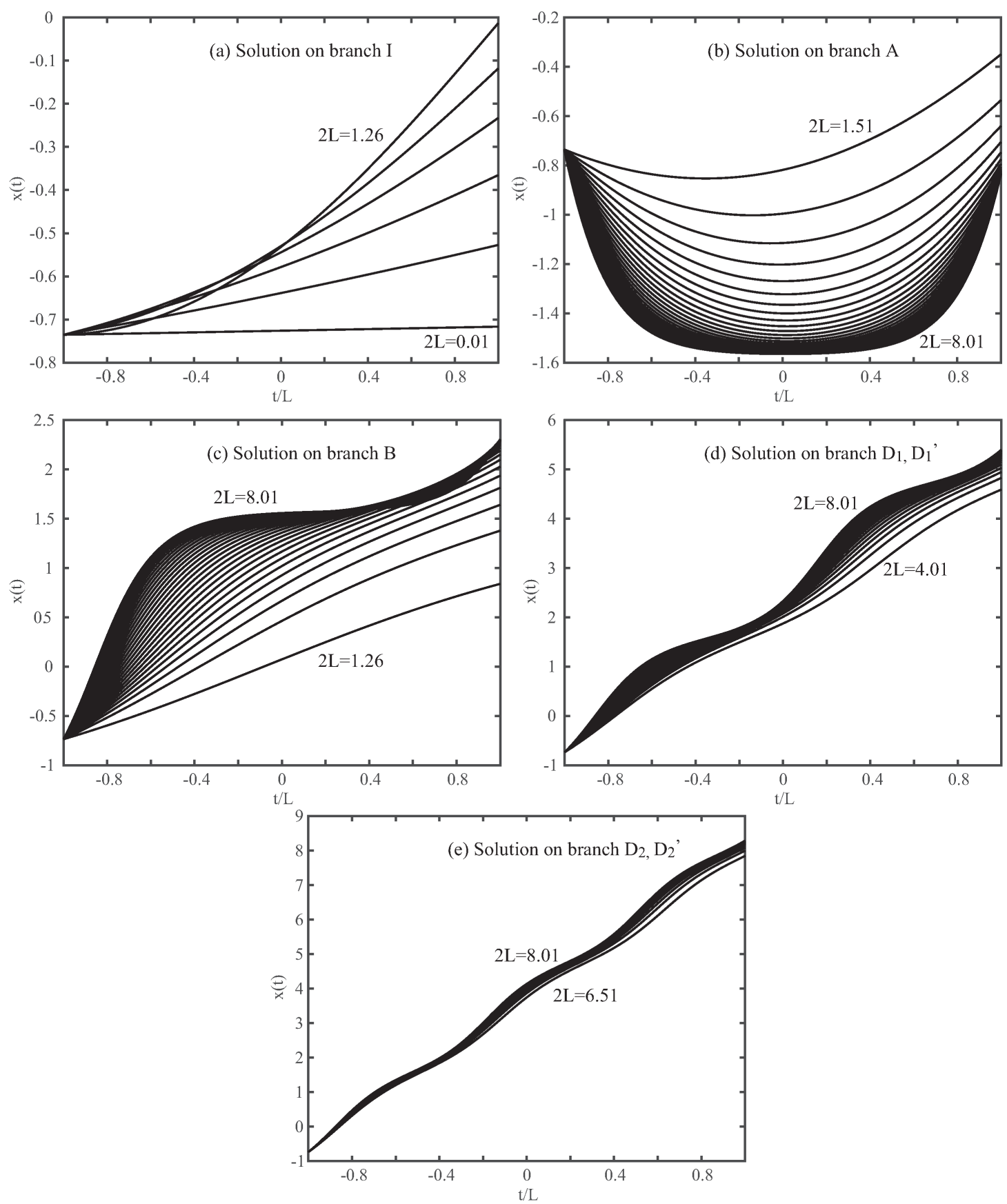

FiguRE 13. Numerically computed solutions $x(t)$ on the various branches in Fig. 12. Values of $2 L$ are in steps of 0.125 between the values indicated.

paper. This type of perturbation analysis could form an interesting future study, based on the starting point described in this paper. 


\section{APPENDIX}

In this Appendix we make some remarks about Conjecture 1.

We recall that the determination of the sign of $\frac{\partial^{2} T_{B}}{\partial z^{2}}(z, \phi)$ directly using (33) seems to be a difficult problem due to the balance between the (positive) integral terms and the nonintegral term that has different signs in different regions of the rectangle $(0,2) \times\left(0, \frac{\pi}{2}\right)$.

Thus, we tried several, less direct, approaches to the convexity of $T_{B}(z, \phi)$. One such approach was the standard method presented in Smoller [22, Chap. 13§D]: to establish the convexity of $T_{B}(z, \phi)$ it is sufficient to prove that

$$
\Phi(z, \phi):=4 \frac{\partial^{2} T_{B}}{\partial z^{2}}+2 k(z, \phi) \frac{\partial T_{B}}{\partial z}>0,
$$

if $(z, \phi) \in(0,2) \times\left(0, \frac{\pi}{2}\right)$ is a stationary point of $z \mapsto T_{B}(z, \phi)$, for all functions $k(z, \phi)$, because at stationary points the value of the first derivative $\frac{\partial T_{B}}{\partial z}$ is zero, by definition. Thus, if we find a function $k(z, \phi)$ such that (47) holds for all points $(z, \phi)$, we conclude that, for each fixed $\phi, \Phi(\cdot, \phi)$ is convex at each of its stationary points, and thus there can exist only one stationary point.

Now, by (27), (33), and (34), choosing

$$
\begin{aligned}
k(z, \phi) & :=-z^{-1}(2-z)^{-1}(1-\cos 2 \phi)^{-1} g(z, \phi) \\
& =(1-\cos 2 \phi)^{-1}-2(z-1) z^{-1}(2-z)^{-1},
\end{aligned}
$$

we get

$$
\Phi(z, \phi)=\left(\int_{0}^{\phi}+\int_{0}^{\bar{x}^{\star}(z)}\right) h^{-5 / 2}(3-k h) d x
$$

where

$$
h=h(z, \phi, x):=z-\cos 2 \phi+\cos 2 x .
$$

From the definition of $k$ it follows that $k<0$ whenever $g>0$ and, from (49), (50), and $h(z, \phi, x)<h(z, \phi, 0)$, we easily conclude that $\Phi(z, \phi)>0$ in $\Omega:=\left\{(z, \phi) \in \mathbb{R}^{2} \mid 3-k(z, \phi) h(z, \phi, 0)>0\right\}$. This set $\Omega$ is illustrated in Figure 14 .

Outside $\Omega$ the sign of $\Phi$ is much harder to establish since the two integrals can have opposite signs and be divergent on the boundaries of the domain of $\Phi$. However, the positivity of $\Phi(z, \phi)$ everywhere in the rectangle $(0,2) \times\left(0, \frac{\pi}{2}\right)$ is easy to get numerically, as illustrated in Figure 15 obtained using the software Mathematica ${ }^{\odot}$.

Alternative approaches to prove the existence of a single minimum of the graph of $z \mapsto T_{B}(z, \phi)$, based on attempting to define different type of time maps via changes of variables [14, 21] or other analytic approaches [14] were fruitless. Proving Conjecture 1 remains a technical challenge.

\section{REFERENCES}

[1] Arakelyan, S.M., Karayan, A.S., Chilingaryan, Yu.S.: Fréedericksz transition in nematic liquid crystals in static and light fields: general features and anomalies. Sov. Phys. Dokl. 29 202-204 (1984).

[2] Belyakov, V.A.: Untwisting of the helical structure in a plane layer of chiral liquid crystal. JETP Lett. 76 88-92 (2002). 


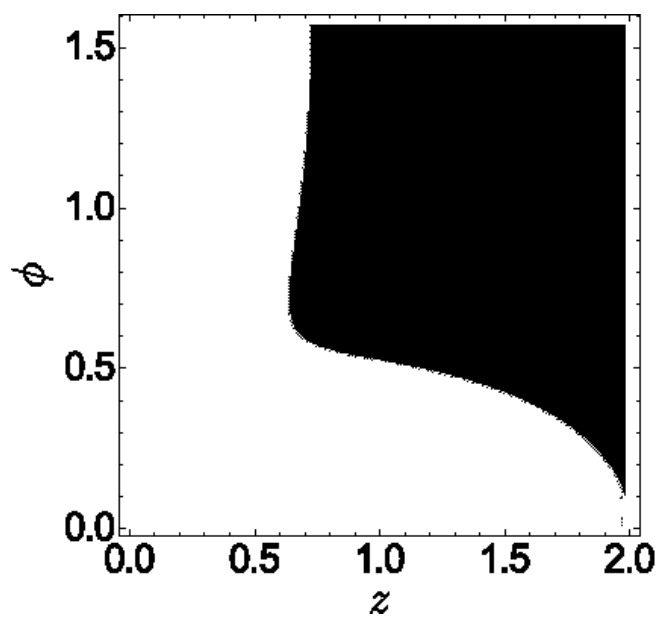

FiguRE 14. Illustration (in black) of the set $\Omega$ of points $(z, \phi)$ where $3-k(z, \phi) h(z, \phi, 0)>0$.

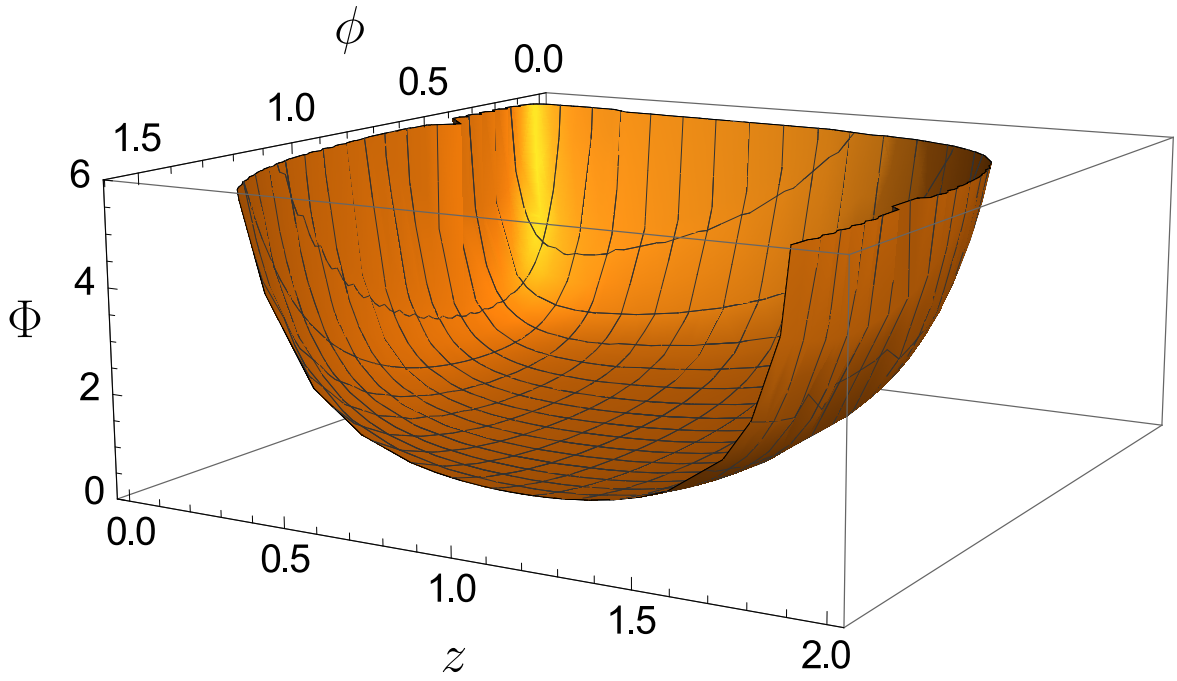

FiguRE 15. Graph of $(z, \phi) \mapsto \Phi(z, \phi)$

[3] da Costa, F.P., Gartland, Jr., E.C., Grinfeld, M., Pinto, J.T.: Bifurcation analysis of the twist-Fréedericksz transition in a nematic liquid-crystal cell with pre-twist boundary conditions. Euro. J. Appl. Math. 20 269-287 (2009)

[4] da Costa, F.P., Méndez, M.I., Pinto, J.T.: Bifurcation analysis of the twist-Fréedericksz transition in a nematic liquid-crystal cell with pre-twist boundary conditions: the asymmetric case. Euro. J. Appl. Math. 28 243-260 (2017)

[5] de Gennes. P.G.: Calcul de la distorsion d'une structure cholesterique par un champ magnetique, Solid State Commun. 6 163-165 (1968). 
[6] de Gennes, P.G. , Prost, J.: The Physics of Liquid Crystals, International Series of Monographs On Physics, vol. 83. Oxford University Press, Oxford (1993)

[7] Deuling, H.J.: Deformation of nematic liquid crystals in an electric field. Mol. Cryst. Liq. Cryst. 19 123-131 (1972).

[8] Dreher, R.: Remarks on the distortion of a cholesteric structure by a magnetic field. Solid State Commun. 13 1571-1574 (1973).

[9] Gartland, Jr., E.C., Huang, H., Lavrentovich, O.D., Palffy-Muhoray, P., Smalyukh, I.I., Kosa, T., Taheri, B.: Electric-field induced transitions in a cholesteric liquid-crystal film with negative dielectric anisotropy. J. Comp. Theor. Nanoscience 7(4) 709-725 (2010).

[10] Gartland, Jr., E.C.: Forces and variational compatibility for equilibrium liquid crystal director models with coupled electric fields. Continuum Mech. Thermodyn. 32 1559-1593 (2020).

[11] Chen, H.-W., Lee, J.-H., Lin, B.-Y., Chen, S., Wu, S.-T. Liquid crystal display and organic light-emitting diode display: present status and future perspectives. Light Sci. Appl. 7 (2018), 17168.

[12] Kedney, P.J., Stewart, I.W.: The untwisting of a bounded sample of cholesteric liquid crystal. Continuum Mech Thermodyn. 6 141-148 (1994).

[13] Kiselev, A.D., Sluckin, T.J.: Twist of cholesteric liquid crystal cells: stability of helical structures and anchoring energy effects. Phys. Rev. E 71031704 (2005).

[14] Korman, P.: Global Solution Curves for Semilinear Elliptic Equations. World Scientific, Singapore (2012).

[15] Lorvanhxay, A.: Time Maps in the Study of Some Boundary Value Problems for Ordinary Differential Equation Problems in the Bounded Interval, (MSc thesis). National University of Laos, Vientiane (2019).

[16] McKay, G.: Unwinding of a cholesteric liquid crystal and bidirectional surface anchoring. J. Eng. Math. 87 19-28 (2014).

[17] Meyer, R.B.: Distortion of a cholesteric structure by a magnetic field. Appl. Phys. Lett. 14 208-209 (1969).

[18] Millar, H., McKay, G.: Director orientation of a twisted nematic under the influence of an in-plane magnetic field. Mol. Cryst. Liq. Cryst. 435 277/[937]-286/[946] (2005).

[19] Sackmann, E., Meiboom, S., Snyder, L.C., Meixner, A.E., Dietz, R.E.: Structure of the liquid crystalline state of cholesterol derivatives, J. Am. Chem. Soc. 90 3567-3569 (1968).

[20] Scarfone, A.M., Lelidis, I., Barbero, G.: Cholesteric-nematic transition induced by a magnetic field in the strong-anchoring model. Phys. Rev. E 84 (2011) 021708.

[21] Schaaf, R.: Global Solution Branches of Two Point Boundary Value Problems, Lecture Notes in Mathematics, vol. 1458. Springer-Verlag, Berlin, Heidelberg (1990).

[22] Smoller, J.: Shock Waves and Reaction-Diffusion Equations, 2nd Ed., Grundlehren der mathematischen Wissenschaften vol. 258. Springer, New York (1994).

[23] Stewart, I.W.: The Static and Dynamic Continuum Theory of Liquid Crystals: A Mathematical Introduction, The Liquid Crystals Book Series 2. Taylor \& Francis, London (2004).

[24] van Sprang, H.A., van de Venne, J.L.M.: Influence of the surface interaction on threshold values in the cholesteric-nematic phase transition. J. Appl. Phys. 57 175-179 (1985) . 
30 F.P. DA COSTA, M. GRINFELD, N. J. MOTTRAM, J.T. PINTO, AND K. XAYXANADASY

Departamento de Ciências e Tecnologia, Universidade Aberta, Lisboa, Portugal, and Centre for Mathematical Analysis, Geometry, and Dynamical Systems, Instituto Superior Técnico, Universidade de Lisboa, Lisboa, Portugal

Email address: fcosta@uab.pt

Department of Mathematics and Statistics, University of Strathclyde, Glasgow, United Kingdom

Email address: m.grinfeld@strath.ac.uk

School of Mathematics and Statistics, University of Glasgow, Glasgow, United KINGDOM

Email address: Nigel.Mottram@glasgow.ac.uk

Departamento de Matemática, Instituto Superior Técnico, Universidade de Lisboa, Lisboa, Portugal, and Centre for Mathematical Analysis, Geometry, and Dynamical Systems, Instituto Superior Técnico, Universidade de Lisboa, Lisboa, Portugal

Email address: jpinto@math.tecnico.ulisboa.pt

Department of Mathematics, Faculty of Natural Sciences, National University of Laos, Dongdok Campus, Vientiane, Lao PDR

Email address: kedtysack@gmail.com 